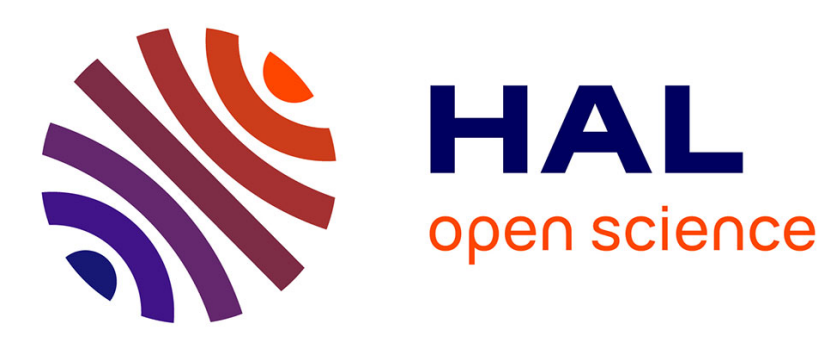

\title{
Introduction : l'édition d'économistes, quel contenu et quelle forme?
}

\author{
Nicolas Rieucau
}

\section{To cite this version:}

Nicolas Rieucau. Introduction: l'édition d'économistes, quel contenu et quelle forme?. Cahiers d'Economie Politique = Papers in political economy, 2009, 57 (2), 10.3917/cep.057.0007 . hal01616986

\section{HAL Id: hal-01616986 \\ https://hal.science/hal-01616986}

Submitted on 15 Oct 2017

HAL is a multi-disciplinary open access archive for the deposit and dissemination of scientific research documents, whether they are published or not. The documents may come from teaching and research institutions in France or abroad, or from public or private research centers.
L'archive ouverte pluridisciplinaire HAL, est destinée au dépôt et à la diffusion de documents scientifiques de niveau recherche, publiés ou non, émanant des établissements d'enseignement et de recherche français ou étrangers, des laboratoires publics ou privés. 


\title{
INTRODUCTION : L'ÉDITION D'ÉCONOMISTES, QUEL CONTENU ET QUELLE FORME?
}

\author{
Nicolas RIEUCAU ${ }^{*}$
}

\begin{abstract}
Résumé :
Comme n'importe quel éditeur scientifique, l'économiste qui entend diffuser les écrits de ses prédécesseurs est confronté à une problématique invariable : publier quoi et comment, par delà le choix de qui. Les difficultés posées par cette problématique, dans le champ de notre discipline, ne se distinguent pas radicalement de celles qui interviennent dans d'autres domaines, y compris en littérature. Certes, le traitement de ces difficultés mobilise à de nombreux égards des compétences qui ne sont habituellement pas l'apanage de l'historien de la pensée économique, mais il requiert aussi des capacités analytiques que ce dernier a coutume de reconnaître comme les siennes propres. Il demeure que l'activité éditoriale est une pratique savante à laquelle l'historien de la pensée économique n'accorde ordinairement que peu d'attention. Pourtant, puisque la littérature primaire dont s'alimente notre discipline est ici en jeu, l'affirmation institutionnelle et la diffusion de celle-ci dépendent d'une telle pratique.
\end{abstract}

\begin{abstract}
Introduction: Editing Economists, what Content and what Form?
Like any other scientific editor, the economist who intends to spread the writings of his predecessors is faced with constant problems, beyond the question of who he will publish: what he should publish and how he should publish it. In the field of our discipline, the difficulties raised by these questions are not radically different from those which occur in other disciplines, including literature. Of course, in many respects dealing with these difficulties implies skills which are not usually the prerogative of the historian of economic thought. Nevertheless it also requires analytical abilities that the latter is in the habit of regarding as being his own. The fact remains that editing is a scholarly practice to which the historian of economic thought usually pays little attention. However, since the primary source materials that continue to be the foundation for research in our academic discipline are here at stake, its institutional recognition and spread depend on such a practice.
\end{abstract}

Mots clefs : Édition, histoire de la pensée économique, édition électronique, appareil critique, texte de base, transcription, traduction

\footnotetext{
* Laboratoire d'Économie Dionysien (Université Paris 8). Courriel : nicolas.rieucau@univ-paris8.fr. Lors de la rédaction de cet article, nous avons pu bénéficier des remarques de $\mathrm{D}$. Besomi, L. Baronian, E. de Champs, G. Dostaler, M.-J. Durand-Richard, J. Hurtado, L. le Maux et A. Orain. Qu'ils en soient ici remerciés. Nous exprimons aussi notre gratitude envers l'ensemble des auteurs du présent numéro qui, outre D. Besomi, ont contribué à l'amélioration de la version finale de ce texte. Bien entendu, aucun d'entre eux n'est responsable des erreurs et des imperfections qui y subsistent.
}

Cahiers d'économie politique, n57, L'Harmattan, 2009, pp. 7-54. 
Keywords: Editing, history of economic thought, electronic edition, critical apparatus, copytext, transcription, translation

Classification du JEL : A 31, B

\section{Introduction}

Depuis bientôt un demi-siècle, l'Université de Toronto organise annuellement un congrès international sur l'édition critique, la Conference on Editorial Problems. Les sujets abordés portent sur des périodes, des thèmes ou des auteurs fort divers 1 . En 1986, ce congrès s'est ouvert, grâce à D. E. Moggridge, à la question de la publication d'économistes tels que Jevons, Marshall, Keynes, Robbins, Meade... (D. E. Moggridge 1988a). Le nombre des participants, s'élevant à 35, a été alors environ deux fois plus faible que celui habituellement rencontré pour les autres éditions, passées ou à venir, de ce congrès ${ }^{2}$. L'initiative de D. E. Moggridge ${ }^{3}$ ne fut d'ailleurs, dans les années qui suivirent, guère imitée. Tandis que les manifestations scientifiques consacrées à l'histoire de la pensée économique se multiplièrent assez considérablement, on le sait, à partir du milieu des années 1980, les difficultés que représente l'édition des textes économiques du passé furent en effet rarement débattues. En la matière, le seul évènement notable fut la conférence organisée par P. Bridel à l'Université de Lausanne en 1991, portant sur les éditions ou les travaux éditoriaux d'économistes comme J.-B. Say, Ferrara, Jevons, Menger, L. Walras, Pareto ou encore Sraffa (P. Bridel 1992a). Depuis cette date, aucune manifestation du même type ne s'est tenue - si l'on s'en tient à celles ayant fait l'objet d'actes publiés - avant celle organisée à Paris, en $2006^{4}$, dont la plupart des contributions font l'objet du présent numéro des Cahiers d'économie politique ${ }^{5}$.

1. Par exemple "Editing Sixteenth-Century Texts" (1965), "Editing Canadian Texts" (1972), "Editing British and American Literature, 1880-1920" (1976), "Editing Poetry from Spenser to Dryden" (1980), "Editing, Publishing, \& Computer Technology" (1984), "Critical Issues in Editing Exploration Texts" (1992), "Editing Robert Grosseteste" (2000), "Editing Philosophers" (2002), "Editing the Bible" (2007). La liste intégrale des sujets traités est disponible à l'adresse http://www.chass.utoronto.ca/cep/. 2. Cette estimation a été établie à partir de l'entrée "Members of the Conference", présente à la fin de la plupart des volumes des actes de ce congrès.

3. Dont le but était précisément, selon ses propres dires, "to reduce the relative isolation of economist editors from each other and from the normal clientele for such meetings" (D. E. Moggridge 1988b, p. 13).

4. «Éditer des textes économiques», Paris, Centre Panthéon, 19-20 mai 2006. Ce colloque a été organisé par le Laboratoire d'Économie Dionysien (Université Paris 8) et le Pôle d'Histoire de l'Analyse et des Représentations Économiques (Université Paris 1).

5. Dans la suite, les renvois relatifs à ces contributions ne consisteront que dans l'indication de leur pagination entre parenthèses, sans mention de l'élément "infra". Signalons que l'intérêt porté par les Cahiers d'économie politique aux questions éditoriales se manifeste aussi par la publication régulière, au sein de sa "Collection", de textes rares ou inédits d'économistes (par exemple de Mirabeau et 
Force est donc de constater qu'en comparaison de leurs homologues œuvrant dans d'autres disciplines, les historiens de la pensée économique ne s'intéressent guère à la question de l'édition ${ }^{6}$. Sans doute est-ce parce qu'ils estiment que ce genre de travail, mal reconnu par leur institution, doit être réservé à l'historien des faits ou au littéraire, ou relève de compétences encore plus exotiques qui sont celles de l'archiviste, du codicologue, du généticien du texte, du paléographe ou encore du textologue ${ }^{7}$. En la matière, les périodiques, les ouvrages ou les manifestations scientifiques où s'expriment tous ces spécialistes sont effectivement nombreux ${ }^{8}$.

Ce qui est en jeu, pourtant, n'est rien d'autre que la publication de la "matière" dont se nourrit notre discipline, c'est-à-dire tout simplement celle du texte qui oriente son cours et sans laquelle, de façon plus essentielle, elle n'existerait pas. Or, pour des raisons principalement commerciales, on assiste ces derniers temps à la diffusion croissante, sous une forme anastatique (plus communément qualifiée de "reprint"), d'éditions anciennes ne comprenant aucun appareil critique, en particulier dans le domaine de l'histoire des idées9. Cette diffusion est également valorisée par les bibliothèques numériques en ligne, certaines d'entre elles comprenant aussi des versions, parfois médiocres, en format texte ${ }^{10}$. Face à ce degré nul ou quasi nul de l'édition savante, il importe de promouvoir des publications de qualité parce que la reconnaissance de l'histoire de la pensée économique en dépend

Quesnay en 1999, Schumpeter en 2005 et Child en 2008).

6. L'absence d'une entrée portant sur cette question dans la classification du Journal of Economic Literature nous semble être aussi une manifestation de cette relative indifférence.

7. Dans la même perspective que la nôtre, R. D. Collison Black (1988, p. 20) indique que le travail éditorial, en particulier, "is a form or aspect of literary scolarship ; as such, it is a type of intellectual activity about which the great majority of modern economists know little and care less, because they do not see it as relevant to their science". R. D. Collison Black (ibid.) ajoute : "It is for this reason that editions of economists' works which incorporate a full scale textual apparatus are comparatively rare". Ce désintérêt pour les matières éditoriales est aussi souligné par P. Dockès et J.-M. Servet (1992, p. 350), A.-M. Fusco (1992, pp. 325-326), A. Jolink (1992, p. 261) ou encore D. E. Moggridge (1992, pp. 365-366). Du reste, comme le rappelle ce dernier à propos de la pensée économique (D. E. Moggridge 1988b, p. 12), "a discipline relatively uninterested in its past is unlikely to place a high value on the production of editions". Signalons que cette négligence est plus marquée en France que dans le monde anglo-saxon. Sur ce point, voir P. Dockès et C. Mouchot (p. 203), J. P. Potier et A. Tiran (pp. 152-153) ainsi que G. de Vivo (2007, pp. 96-97). Cela étant, à la lecture d'A. Grésillon (1994, pp. 186-187), on constate que cette dernière disparité se manifeste également dans le domaine de l'édition littéraire.

8. Pour s'en faire une idée, dans le domaine de la recherche anglo-saxonne, consulter la bibliographie dressée par W. Baker et K. Womack (2000, notamment part. IV), complétée en partie par celle du Committee on Scholarly Editions (2006, pp. 39-46).

9. Sur ce point, voir T. Aspromourgos (2000, pp. 2-3). J. P. Potier et A. Tiran (p. 152, n. 1) font aussi allusion à ce phénomène.

10. Nous ne nions pas que les serveurs en question (Les classiques des sciences sociales, Gallica, Google Books, Project Gutenberg, Text Archive, The Making of the Modern World: The Goldsmiths'Kress Library of Economic Literature, Wikisource...) puissent être d'un certain secours. Mais, outre le fait que ces serveurs ne comportent pour la plupart, compte tenu des droits d'auteur, que des éditions datées au mieux d'un demi-siècle, il arrive que celles-ci soient numérisées de façon fautive ou lacunaire. 
et, en conséquence, de s'interroger sur ce que doivent être leurs principales caractéristiques.

Les contributions issues du colloque organisé en 2006 reflètent, dans leur majorité, une dimension particulière de l'objet éditorial et de sa problématique en matière d'histoire de la pensée économique. Du point de vue de l'objet, les textes publiés ci-après ${ }^{11}$ se concentrent sur l'édition de l'œuvre d'une douzaine d'économistes de diverses époques (notamment Quesnay, Bentham, Sismondi, L. Walras, Pareto, Harrod et Sraffa), et non pas sur des courants (les socialistes, les anarchistes, l'école historique allemande, les keynésiens, les nouveaux classiques...) ou des thèmes (les techniques, les crises financières, la guerre, le chômage...) précis. La problématique associée à cet objet, quant à elle, porte essentiellement sur la question de savoir quoi publier et comment, et non sur le choix de qui. Cette dernière interrogation, pour fondamentale qu'elle soit, s'inscrit au sein d'un questionnement plus général, et maintes fois débattu, sur la fonction de l'histoire de la pensée économique. En revanche, la réflexion relative au contenu (publier quoi) (Première partie) et à la forme (publier comment) (Seconde partie) de l'édition n'est justement guère traitée par les historiens de la pensée économique.

En développant ici cette réflexion ${ }^{12}$ et en essayant par là même d'ébaucher un modeste vade-mecum de l'économiste-éditeur ${ }^{13}$, nous entendons démontrer deux choses :

- Que le travail éditorial en histoire de la pensée économique ne présente pas de spécificités suffisantes, en comparaison d'autres champs disciplinaires, pouvant justifier le désintérêt dont il souffre.

- Que la frontière entre édition et interprétation est plus floue que l'on pourrait le croire ${ }^{14}$.

\footnotetext{
11. À leur suite, sont reproduits et présentés un ou plusieurs manuscrits inédits, ce dont nous nous réjouissons particulièrement.

12. Un chapitre spécifique ("Appendice", infra, pp. 37-40) est consacré au support électronique comme moyen de résoudre plusieurs problèmes mis en évidence par cette réflexion.

13. Pour illustrer notre propos, nous nous appuierons principalement sur les contributions du présent recueil ainsi que sur celles qui y sont associées. Par exemple, il sera fait référence au commentaire cijoint (pp. 197-210) des Euvres Économiques Complètes (1987-2005) des Walras mais aussi à l'ensemble des contributions, publiées par ailleurs, relatives à cette édition. Les autres éditions ou contributions citées relèveront de l'histoire de la pensée économique ou d'autres domaines.

14. Ce point est notamment revendiqué de façon explicite, dans le présent volume, par C. Théré et L. Charles (p. 68). Rappelons que la question de savoir qui publier, que nous avons écartée, s'inscrit évidemment dans un registre interprétatif.
} 


\section{Première partie. Publier quoi ?}

La question de la délimitation de ce que pourrait constituer les "œuvres complètes" d'un écrivain, qu'il soit économiste ou non, n'est pas souvent évidente ${ }^{15}$ (1. 1.). Il est certes plus facile de déterminer ce qui relève des "œuvres complètes" d'un Radiguet ou d'un Cantillon que de celles d'un Voltaire ou d'un Marx mais, de manière générale, parmi toutes les traces écrites laissées par un auteur au moment de sa mort, le choix de celles devant être considérées comme relevant à proprement parler de son œuvre véhicule inévitablement une part d'arbitraire. Cette part d'arbitraire se constate aussi, cela va peut-être davantage de soi, lorsque l'on souhaite publier une édition sélective. Dans ce second cas, la délimitation du corpus peut toutefois être également influencée par le poids plus ou moins important de certaines contraintes objectives (1. 2.). Enfin, qu'une édition se présente comme une publication complète ou sélective, il existe plusieurs autres difficultés relatives à l'établissement de son contenu (1.3.).

\section{1. Qu'est-ce qu'une édition complète ?}

"Quand on entreprend de publier, par exemple, les œuvres de Nietzsche, où faut-il s'arrêter? Il faut tout publier, bien sûr, mais que veut dire ce "tout" ? Tout ce que Nietzsche a publié lui-même, c'est entendu. Les brouillons de ses œuvres ? Évidemment. Les projets d'aphorismes ? Oui. Les ratures également, les notes au bas des carnets ? Oui. Mais quand, à l'intérieur d'un carnet rempli d'aphorismes, on trouve une référence, l'indication d'un rendezvous ou d'une adresse, une note de blanchisserie : œuvre, ou pas œuvre? Mais pourquoi pas ? Et cela indéfiniment", Foucault (1969, p. 794).

Ce passage d'une fameuse conférence donnée par Foucault à la fin des années soixante - époque où il venait d'éprouver les affres de la publication des Euvres philosophiques complètes de Nietzsche - devant la Société française de philosophie, trouvera un écho humoristique, quelques années plus tard, lorsque W. Allen annoncera la publication du Recueil des listes de blanchissage de Hans Metterling ${ }^{16}$.

15. Il n'existe pas, à notre connaissance, d'étude exhaustive consacrée à cette notion. On trouvera cependant quelques éléments dans J. Sgard \& C. Volpilhac-Auger (1999).

16. En voici quelques extraits : "Vénal et fils viennent enfin d'éditer le premier volume, longtemps attendu, des listes de blanchissage de Metterling (Recueil des listes de blanchissage de Hans Metterling, tome 1, 437 pages et une introduction de 32 pages, plus un index : \$18,75) agrémentées de commentaires érudits par le fameux metterlinguiste Gunther Eisenbud.

[...] À la vérité, la toute première liste de blanchissage Metterling :

Liste $\mathrm{n}^{\circ} 1$

6 caleçons

4 gilets de corps

6 paires de chaussettes bleues

4 chemises bleues

2 chemises blanches

6 mouchoirs

Pas d'amidon 
Néanmoins, par delà la parodie que l'on peut en faire, le cas des notes de blanchisserie est-il si caricatural que cela si l'on entreprend de publier, par exemple, un auteur du XVIII ${ }^{\mathrm{e}}$ siècle ? On sait en effet que les écrivains des Lumières portaient des grandes chemises à volant, fort coûteuses à entretenir : de ce point de vue, n'estil pas piquant de savoir que Rousseau eut avant sa "réforme somptuaire", comme il le reconnaît dans ses Confessions (1770, p. 364), "la passion du beau linge" et supporta en conséquence des frais de blanchisserie astronomiques, ou encore que Diderot épousa une lingère (Antoinette Champion), peut-être pas tout à fait par hasard ${ }^{17}$ ?! Enfin, si les dépenses de blanchisserie et autres notes domestiques de ces derniers ne sont en réalité pas parvenues jusqu'à nous, on remarquera que celles d'autres auteurs, fort divers, ont été incorporées au sein de leurs œuvres complètes. On en trouve ainsi dans les corpus respectifs de Galilée - Ricordi autografi (15981634) - ou encore de Baudelaire - Carnet (1861-1863). Pourquoi n'en serait-il pas de même pour les dépenses d'un économiste, a fortiori si l'on considère que les informations biographiques le concernant peuvent éclairer son œuvre intellectuelle?

On sait toutefois que ce point est débattu ${ }^{18}$ et, de manière plus générale, l'exemple des notes de blanchisserie révèle combien la détermination de la complétude d'une œuvre peut être difficile. De prime abord aisé à définir, le désir de "tout publier" ne l'est pas. Il introduit des éléments interprétatifs propres à

constitue une introduction parfaite, quasi totale, à ce génie inquiet, surnommé par ses contemporains "le Fantastique Pragois".

Cette liste fut griffonnée à la hâte à l'époque où Metterling écrivait Les confessions d'un fromage anormal. [...] La répugnance de Metterling pour l'amidon est typique de cette période, et quand son premier paquet de linge lui revint trop raide, Metterling se sentit triste et déprimé.

[...] Ce qui empêcha Metterling de mener à bien son recueil de poèmes pourtant si longuement mûri fut une histoire d'amour avortée, qui se lit en filigrane dans la célèbre "quatrième liste" :

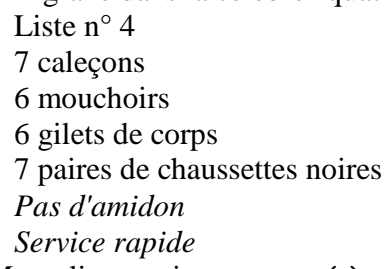

[...] Manifestement, la personnalité de Metterling avait commencé à se détériorer dès 1894, si nous pouvons nous fier à la sixième liste :

$$
\begin{aligned}
& \text { Liste } \mathrm{n}^{\circ} 6 \\
& 25 \text { mouchoirs } \\
& 1 \text { gilet de corps } \\
& 5 \text { caleçons } \\
& 1 \text { chaussette }
\end{aligned}
$$

et il n'y a rien d'étonnant à apprendre que ce fut à cette époque que commencèrent ses séances de psychanalyse avec Freud." ; Allen (1972, pp. 7-13).

17. Comme le souligne A. M. Wilson (1972, p. 33), dans sa biographie du co-éditeur de l'Encyclopédie.

18. Pour un commentaire, voir notamment D. A. Walker (1983). 
l'élaboration inévitable, mais plus ou moins consciente et affichée, de ce que Foucault (1969, p. 794) nomme la "théorie de l'œuvre". Et, comme ce dernier le suggère, les exemples de cas difficiles à trancher, aussi hétéroclites soient-ils, peuvent être multipliés. Nous pensons aux cahiers d'écolier, aux textes d'attribution incertaine, aux écrits rédigés ou signés au nom d'une institution, aux comptes rendus de réunions, aux traductions effectuées par l'auteur, aux marques "muettes" que constituent les traits tirés en marge ou en interligne des textes d'autres auteurs, aux notes de lecture, aux inscriptions dites "de régie" relatives par exemple à la numérotation d'un feuillet, à la localisation d'un document dans l'organisation générale d'un projet intellectuel ou au degré de satisfaction que l'auteur éprouve quant à l'achèvement de son manuscrit, etc. : tous ces éléments doivent-ils être considérés comme faisant partie du corpus intégral des œuvres de l'auteur ${ }^{19}$, qu'il soit économiste ou non?

\section{2. Qu'est-ce qu'une édition sélective?}

La difficulté que représente la détermination d'une édition sélective nous paraît se présenter de façon prononcée dans deux cas précis. Tout d'abord si l'on envisage de publier les écrits "économiques" d'un auteur antérieur au début du XIX ${ }^{\mathrm{e}}$ siècle, période à laquelle l'autonomisation de notre discipline a commencé de s'affirmer. On devine alors, en effet, que la circonscription de ces écrits est épineuse, quoiqu'une telle difficulté puisse continuer de se manifester si l'on entend éditer l'œuvre économique de certains auteurs des $\mathrm{XIX}^{\mathrm{e}}$ et $\mathrm{XX}^{\mathrm{e}}$ siècles dont la pensée est irriguée par des réflexions issues d'autres champs disciplinaires (1.2.1.). En outre, quelle que soit l'époque à laquelle appartient l'auteur, les choix de l'économiste éditeur sont conditionnés par sa démarche épistémologique, c'est-à-dire par la manière dont il conçoit ce que doit être la bonne pratique de l'histoire de la pensée économique elle-même (1. 2. 2.). Plusieurs contraintes objectives exercent enfin un rôle sur la détermination d'une édition sélective. À l'instar de ses homologues publiant des œuvres relevant d'autres disciplines, l'économiste éditeur voit ses décisions relatives au contenu de la publication qu'il envisage d'effectuer dépendre plus précisément de trois éléments. Premièrement, l'histoire de l'édition ; deuxièmement les choix et les pratiques éditoriales de l'auteur lui-même; troisièmement les aspects financiers et matériels ${ }^{20}(1.2 .3$.).

19. En excluant le fait que ces éléments puissent détenir indirectement une valeur informative. Si par exemple l'un d'entre eux est daté, l'analyse de son support ou la connaissance de son instrument d'écriture peut suggérer la période de rédaction d'un texte ayant le même type de caractéristiques matérielles.

20. Certains de ces aspects peuvent évidemment avoir partie liée avec l'histoire de l'édition. Toutes choses restant égales par ailleurs, un texte inédit bénéficiera de meilleures ventes qu'un texte maintes fois publié dans le passé. 


\section{2. 1. Sélection de l'œuvre et autonomisation de la pensée économique}

Si on la compare par exemple à la pensée politique et plus encore à la pensée philosophique ou mathématique, la pensée économique est une discipline assez jeune, dont on considère d'habitude qu'elle n'a commencé à être reconnue institutionnellement qu'au début du XIX ${ }^{\mathrm{e}}$ siècle. Cette époque est marquée par la naissance des sociétés d'économie politique et d'un véritable lectorat, les débuts de son enseignement, la multiplication des périodiques, des dictionnaires et des manuels qui lui sont consacrés ${ }^{21}$. En somme, ce n'est qu'à l'époque de Ricardo en Angleterre et de Say en France que la pensée économique serait devenue véritablement autonome: entreprendre une édition sélective axée sur les écrits économiques d'un auteur antérieurs à cette époque, en supposant qu'il soit possible de singulariser de tels écrits ${ }^{22}$, ne pourrait se faire sérieusement sans publier aussi un certain nombre de textes, de nature non-économique, qui participent de leur intelligibilité. Il serait dans ce cas assez malaisé de ne pas verser dans l'arbitraire lors du choix de ces textes conjoints.

Cette difficulté peut être facilement illustrée, y compris si l'on prend l'exemple d'écrits économiques ne précédant que de peu l'avènement de la pensée économique en tant que discipline autonome. Prenons le cas des textes de Hume. En admettant, contrairement à la tradition schumpeterienne ${ }^{23}$, que ceux ayant un rapport avec l'économie ne se limitent pas, pour l'essentiel, à certains Discours politiques (1752, 1760), c'est alors presque tout le reste de l'œuvre - à l'exception de l'Histoire d'Angleterre (1754-1762) et d'une partie de la correspondance - qui s'offre à l'économiste éditeur. Et, si plusieurs Essais moraux et politiques $(1741,1742)$ ainsi que le "Livre II" (1739) du Traité de la nature humaine s'imposent assez aisément, la sélection demeure plus difficile et nécessairement arbitraire pour d'autres écrits, à commencer par ceux relevant directement de sa philosophie morale et politique. Cette difficulté intervient aussi, par exemple, s'il est question de choisir, dans le cadre de la publication des œuvres économiques de Rousseau, le corpus devant accompagner le Discours sur l'économie politique $(1755,1758)$. L'étendue de ce corpus dépend en effet de la façon dont on apprécie Rousseau en tant qu'économiste. Si le Citoyen de Genève est envisagé comme un auteur rétrograde, en particulier peu au fait de la "Science nouvelle" des physiocrates, l'intérêt de lire et donc de publier - ses écrits économiques sera cantonné à un domaine purement historique, comme un témoignage d'idées déjà surannées à son époque, axées sur la

21. Sur ces différents points, on peut consulter A. Béraud (1992, pp. 306-308), J. Hecht (1989, pp. 4675), É. Laurent \& L. Marco (1996, pp. 79-82), L. Levan-Lemesle (1986), J. A. Schumpeter (1954, t. I, pp. 13-14).

22. Ce qui ne serait bien entendu pas le cas si l'on souhaitait publier, par exemple, les passages économiques des Politiques et de l'Éthique à Nicomaque d'Aristote, ou encore ceux de la Somme Théologique de Thomas d'Aquin.

23. J. A. Schumpeter (1954, t. I, p. 179, n. 1). Sur ce dernier et les commentateurs qui partagent son point de vue, voir par exemple D. Deleule (1979), p. 80, n. 9. 
revendication du modèle économique, fondamentalement dépassé, de l'autarcie et de la petite exploitation agricole. La publication, si tant est qu'elle soit projetée, serait alors assez succincte. Outre le Discours sur l'économie politique, elle se limiterait à des extraits de quelques textes importants : extraits du Discours sur les sciences et les arts (1750), du Discours sur l'origine et les fondements de l'inégalité (1755), du Projet de Constitution pour la Corse (1760-1769) et des Considérations sur le gouvernement de Pologne (1771). Cette frilosité éditoriale n'existerait pas si l'on adhérait, en revanche, à la figure d'un Rousseau économiste critique, réprouvant sur une base analytique solide la perte de liberté associée au développement de la société commerciale et ayant une pensée alternative à la pensée "libérale" de son temps - pensée alternative mettant en valeur, de manière cohérente, une économie agricole non marchande source d'abondance. L'idée même de publier Rousseau serait alors mieux enracinée, car justifiée d'un point de vue historique mais aussi analytique, et le choix des textes colligés nettement plus ouvert - incluant par exemple certaines lettres de La Nouvelle Héloïse (1760) - chacun d'entre eux participant à la constitution d'une théorie considérée comme aboutie ${ }^{24}$.

Un tel problème de délimitation du corpus pourrait être illustré par de nombreux autres écrits, plus tardifs encore, du XVIII siècle. On devine qu'il intervient également, par exemple, s'il s'agit de déterminer l'étendue des textes relevant de l'œuvre économique de Condillac ou de Smith : quels seraient les écrits qu'il conviendrait d'associer respectivement à Le commerce et le gouvernement $(1776)^{25}$ et à la Richesse des nations $(1776)^{26}$ ?

Cela étant, il est discutable de considérer qu'un tel problème de circonscription

24. On trouvera dans B. Bernardi (2002, part. I et III), quelques éléments opposant ces deux lectures de Rousseau. Notons que si la seconde d'entre elles est privilégiée, l'élargissement conséquent du corpus pourrait toutefois conduire, paradoxalement, à ne plus le qualifier d' "économique" dans la mesure où les extraits choisis s'inscriraient dans un cadre théorique relativement vaste, dérivant à proprement parler de la "philosophie sociale" de Rousseau.

25. Estimant que le seul intérêt de cet ouvrage consiste dans la théorie de la valeur-utilité qui y est exposée, une lecture marginaliste privilégierait tout ou partie de certains textes philosophiques, à commencer par le Traité des sensations (1754) et le Traité des animaux (1755). Cette lecture marginaliste a été développée par J. Marchal (1938) et É. Morand (1912). Plus récemment, W. Eltis (1995) s'en est rapproché. En revanche, parce qu'elle souligne l'unité et la nature totalisante du projet intellectuel de Condillac, une lecture que l'on pourrait qualifier de systémique, que l'on trouve par exemple chez P. Tort (1982) et surtout chez A. Bertrand (1998) et A. Orain (2003) et (2004), se refuserait à toute édition sélective sur la base du seul critère "économique".

26. Ayant débuté à l'époque de la publication des premiers volumes des Works and Correspondence (1976-1978) de Smith, il y a une trentaine d'années, la relecture et la réévaluation de la pensée économique du professeur de Glasgow ont conduit à envisager celle-ci, on le sait, sans se limiter à la Richesse des nations. Deux autres textes majeurs ont été en particulier sollicités: la Théorie des sentiments moraux (1759) et les Leçons sur la jurisprudence (1762-1763, 1766). Comme pour Condillac, cette lecture pour ainsi dire compréhensive, privilégiée par exemple par M. Biziou (2003), n'admettrait pas l'idée d'une sélection de textes de Smith uniquement fondée sur un critère "économique". 
ne se posera plus par la suite, plus précisément à partir du début du XIX ${ }^{\mathrm{e}}$ siècle, époque à laquelle la pensée économique fait figure de champ de connaissance ayant sa logique propre, comme l'illustre l'analyse ricardienne. Si son développement sui generis ne saurait être nié, la pensée économique continuera en effet de se nourrir par ailleurs, rappellent P. Dockès et J.-M. Servet (1992, p. 346), des travaux des gestionnaires, des ingénieurs ou des mathématiciens dont la publication de l'œuvre strictement "économique" ne serait pas sans difficultés. Ces problèmes de délimitation du corpus "économique" peuvent aussi être évoqués pour des économistes des $\mathrm{XIX}^{\mathrm{e}}$ et $\mathrm{XX}^{\mathrm{e}}$ siècles dont l'œuvre a partie liée avec la philosophie, qu'il s'agisse de J. S. Mill, Marx, Menger, Keynes, Hayek ou encore Sen, pour ne citer que quelques auteurs majeurs.

Au demeurant, ces difficultés éditoriales peuvent, en retour, se manifester pour des auteurs œuvrant dans des disciplines considérées en quelque sorte comme canoniques, telles que les mathématiques et la philosophie ${ }^{27}$. En somme, la classification des sciences à une époque donnée et l'affirmation institutionnelle de tel ou tel de ses éléments ne doivent pas masquer le fait que l'œuvre de nombreux auteurs, qu'ils soient ou non économistes, n'est compréhensible que s'il est fait abstraction des frontières disciplinaires. Publier leurs écrits en privilégiant un domaine spécifique et pour ainsi dire "officiel" de la connaissance sera souvent, en ce sens, une entreprise soumise à l'arbitraire.

\section{2. 2. La démarche épistémologique de l'éditeur comme critère de ses choix}

S'il s'agit de publier des écrits relevant d'une discipline étroitement liée à la réflexion historique, comme peut l'être la pensée économique, la décision relative au contenu du corpus peut dépendre en partie de la façon dont on envisage la pratique correcte de cette réflexion elle-même. Trois approches peuvent être distinguées ${ }^{28}$. Lorsque la démarche est rétrospective, les textes auront tendance à être sélectionnés en fonction de leur capacité à préfigurer la pensée économique ultérieure. Si elle est extensive, la détermination du corpus sera peut-être moins restrictive. Elle sera orientée par la manière de rendre compte du contexte intellectuel ou factuel dans lequel s'inscrit la pensée économique de l'auteur. Quand, enfin, la démarche est intensive, on s'efforcera de mettre l'accent sur les textes

27. Prenons le cas d'une édition des "œuvres mathématiques" de certains auteurs des $\mathrm{XVI}^{\mathrm{e}}$ et $\mathrm{XVII}{ }^{\mathrm{e}}$ siècles. Tandis que les écrits mathématiques de Viète, le fondateur de l'algèbre, sont assez aisés à délimiter, il n'en va pas de même de ceux de Descartes et de Pascal, parce qu'ils font souvent corps avec leur pensée philosophique. Si l'on prend l'exemple de mathématiciens du XIX e siècle, la même conclusion s'impose : l'œuvre mathématique d'Abel ou de Galois n'accorde guère de place aux considérations philosophiques, ce qui n'est absolument pas le cas des écrits de Cournot - auteur dont la pensée économique elle-même, soit dit en passant, est assez difficile à circonscrire parce que également imprégnée de considérations mathématiques et philosophiques -, de Cayley ou encore de Clifford.

28. Nous reprendrons ici la terminologie proposée par A. Lapidus (1995). 
considérés comme enrichissants pour l'analyse économique moderne.

Il est aisé d'illustrer ce point à partir des éditions commentées ou présentées dans ce numéro des Cahiers d'économie politique. Comme l'indique N. Sigot (pp. 104-105), la première de ces démarches a conditionné les choix opérés par W. Stark dans son édition des Jeremy Bentham's Economic Writings (1952-1954). En revanche, N. Sigot elle-même a privilégié la démarche extensive : ses choix relatifs aux Euvres économiques (1787-1801) de Bentham ont été principalement déterminés par le désir de replacer la pensée de ce dernier dans le cadre juridique et législatif qui la dépasse et la conditionne (pp. 105-106), non sans prendre en compte la question de l'intervention économique de l'État concernant la gestion de la pauvreté (pp. 106-107), ardemment débattue au début du XIX ${ }^{\mathrm{e}}$ siècle en Angleterre. M. Dal-Pont Legrand et L. Frobert, quant à eux, déclarent qu'ils ont opté pour une position médiane lors de leur sélection des Écrits économiques de Juglar. S'ils ont retenu ses travaux sur les cycles, lesquels lui ont valu sa reconnaissance ultérieure parmi les économistes, leur choix s'est toutefois élargi à ses textes relatifs à la monnaie d'une part et à la population d'autre part, non seulement parce que ces deux catégories de textes sont associés à sa pensée sur les cycles (p. 184-185), mais aussi parce que "[c]es trois séries de travaux liés chez Juglar (crises, monnaie, population) traduisent nombre de connexions avec l'environnement intellectuel de son temps" (p. 185). Tout en ne négligeant pas la démarche extensive, la politique éditoriale dont $\mathrm{P}$. Bridel rend compte s'est néanmoins également ouverte à la perspective intensive dans la mesure où la pensée de Sismondi lui semble d'actualité (pp. 136-137, 140-141, 145), tant du point de vue épistémologique lorsqu'elle envisage les rapports entre notre discipline et les autres sciences humaines, qu'au regard de ses objets, comme le développement, la pauvreté ou les inégalités.

\section{2. 3. Les contraintes objectives}

Les contraintes objectives qui pèsent sur la détermination du corpus peuvent pareillement être illustrées par les contributions dont il est fait état dans le présent recueil. L'histoire de l'édition ainsi que les aspects financiers et matériels ont par exemple pesé sur les choix effectués par les éditeurs de Quesnay. L. Charles et C. Théré (p. 83, n. 42) ${ }^{29}$ rappellent en effet qu'ils ont écarté certaines collaborations entre Quesnay et Mirabeau, telles que le Bref état des moyens pour la restauration de l'autorité du roi et de ses finances (1757-1760) et le Traité de la monarchie (1757-1759), parce qu'elles avaient déjà été diffusées, respectivement par G. Weulersse en 1913 et G. Longhitano en 1999. En outre, si la nécessité d'une édition des Euvres économiques du docteur s'imposait d'autant plus que le recueil François Quesnay et la physiocratie (1958) était épuisé, l'ampleur de la nouvelle édition a été cependant limitée par des contraintes de temps et de place. C'est ainsi que seule la version imprimée - et non les trois versions manuscrites - du Tableau économique

29. Voir aussi C. Théré, L. Charles et J.-C. Perrot (2005a, pp. xxvi-xxvii). 
avec ses explications (1760) a été publiée (p. 77). De façon plus générale, l'élaboration d'une édition vraiment exhaustive des écrits économiques de Quesnay, y compris de ses interventions sur les manuscrits de Mirabeau, a été écartée car elle aurait pris "plusieurs dizaines d'années" (p. 75) ; l'essentiel étant dès lors de confectionner une édition non pas parfaite, mais perfectible (p. 77, n. 25)

À des degrés divers, les deux contraintes précédemment énoncées, auxquelles s'ajoute celle due aux choix et aux pratiques éditoriales de l'auteur lui-même, se sont quant à elles manifestées lors de la préparation des Euvres économiques (17871801) de Bentham. D'une part, en effet, la décision de publier la Défense de l'usure (1787), l'Esquisse d'un ouvrage en faveur des pauvres (1802) et le Manuel d'économie politique (1811) a été motivée par la non-parution, depuis le $\mathrm{XIX}^{\mathrm{e}}$ siècle, d'une version en français de ces écrits (p. 108) ; tandis que la diffusion dans cette langue de l'œuvre du juriste anglais constitue une véritable tradition éditoriale avec laquelle il s'agissait précisément de renouer (pp. 115-116). D'autre part, Bentham lui-même a reconnu la qualité des traductions de Dumont, retenues pour l'édition (p. 117). Enfin, des raisons financières ont notamment déterminé l'exclusion des annotations de Ricardo en marge du manuscrit Sur les prix (1797-1801) de Bentham - l'autre raison principale étant l'existence de deux publications antérieures (réalisées par Silberner en 1939 puis Sraffa en 1951) de ces annotations (p. 115).

Il est aussi possible de renvoyer au corpus Walras, où seules les contraintes financières et matérielles ne semblent pas avoir joué de rôle déterminant. P. Dockès et C. Mouchot (p. 200) ${ }^{31}$ rappellent ainsi que la correspondance d'A. Walras a été publiée parce qu'elle était inédite, tandis que celle de L. Walras a en revanche été exclue compte tenu de l'existence de l'édition, de grande qualité, réalisée par W. Jaffé dans les années 1960. Par ailleurs, P. Dockès et C. Mouchot (p. 199) expliquent qu'ils ont respecté le souhait de L. Walras en décidant de joindre ses œuvres économiques à celles de son père.

\section{3. Problèmes communs à la détermination du contenu d'une édition complète ou sélective}

Quelles que soient l'ampleur et l'inscription disciplinaire de la publication entreprise, son établissement nécessite parfois un travail, relativement conséquent, de repérage, de dépouillement et de prospection des pièces qui seront finalement publiées. Cet accès aux sources peut aussi intervenir plus tardivement dans le

30. Se reporter également à C. Théré, L. Charles et J.-C. Perrot (2005a, p. xix) et L. Charles, J.-C. Perrot et C. Théré (2009, p. 93). Même s'il évoque ces contraintes matérielles, G. de Vivo (2009, p. 102) semble toutefois les avoir sous-estimées.

31. Voir aussi P. Dockès \& alii (1990, p. xli).

32. Voir également P. Dockès \& alii (1990, p. xl). 
processus de préparation de l'édition, lorsqu'il s'agit de vérifier par exemple la transcription d'une pièce manuscrite ou d'essayer de la dater (1.3. 1.). Ce genre de recherches, qui valent à l'éditeur (économiste ou non) d'être parfois comparé à un véritable détective, peuvent en outre être compliquées par la nécessité d'identifier le nom des auteurs dissimulés derrière l'anonymat ou le pseudonymat, d'attribuer la part qui revient à chacun quand on est confronté à un écrit rédigé collectivement, ou encore lorsqu'il s'agit d'attester l'authenticité d'un texte (1. 3. 2.). Deux autres difficultés, enfin, sont communes à la plupart des éditions complètes ou sélectives : la détermination du texte de base (1.3. 3.) et celle des diverses caractéristiques de l'appareil critique (1.3. 4.).

\section{3. 1. Accéder aux sources}

Depuis les années 1980, l'accession aux sources est facilitée par l'existence de catalogues électroniques de plus en plus préci ${ }^{33}$. De surcroît, avec le développement du réseau Internet, au milieu de la décennie suivante, certains de ces catalogues ont été progressivement mis en ligne ${ }^{34}$. De la sorte, il est parfois possible de repérer, à distance, la présence d'un texte rare ou inédit en quelques minutes, tandis que cette information n'aurait pu être auparavant recueillie qu'en sollicitant les services d'une personne (collègue ou conservateur) susceptible de parvenir aisément au catalogue ou au fonds, à moins qu'il n'eût été nécessaire de se déplacer soi-même physiquement pour dépouiller celui-ci. Les "spatial difficulties" illustrées par P. Groenewegen (1992, pp. 16-18) ont été donc relativement réduites depuis une trentaine d'années ${ }^{35}$.

Pour autant, elles ne sont pas devenues insignifiantes. Les instruments de recherche électroniques présents sur la Toile ne sont réellement développés que pour les fonds conservés aux États-Unis et, dans une mesure légèrement moindre, en Europe. Ils sont, en particulier, quasi inexistants pour les fonds des Pays de $l^{\prime} E s t^{36}$. En conséquence, une prospection dans les catalogues sur format papier et non numérisés s'impose souvent. Par ailleurs, il arrive qu'un dépouillement effectif d'un fonds soit nécessaire, parce que les inventaires disponibles demeurent

33. Rappelons que les atouts de l'électronique, concernant la façon dont l'édition se présente, relativement à son contenu et à sa forme, sont discutés dans 1' "Appendice", infra, pp. 37-40.

34. Au sujet des fonds manuscrits et pour se limiter au cas de la France, signalons la mise en ligne récente des catalogues de la Bibliothèque Nationale de France (http://archivesetmanuscrits.bnf.fr/) et des Établissements de l'Enseignement Supérieur (http://www.calames.abes.fr/pub/).

35. Lorsqu'elle est autorisée, la reproduction de documents est pareillement facilitée aujourd'hui par le progrès des techniques. Souvent, grâce au développement du numérique, l'éditeur n'est par exemple plus contraint de commander la confection de la microforme d'un volume entier de manuscrits (alors que seuls quelques-uns d'entre eux l'intéressent!) ou de supporter des frais importants de photographies.

36. Pour le chercheur dix-huitièmiste, le guide récemment paru (G. Dulac et S. Karp 2007) des archives des pays de l'Empire russe, selon son territoire de la fin du XVIII ${ }^{\mathrm{e}}$ siècle (en exceptant la Pologne dans ses frontières actuelles), est à ce titre particulièrement précieux. 
insuffisamment détaillés ${ }^{37}$ ou carrément absents. En outre, même si la pièce recherchée est déjà identifiée, il faut parfois la consulter sur place, lorsque sa reproduction est interdite, pour en vérifier la transcription (s'il s'agit d'un manuscrit) ou encore, à des fins de datation, pour en analyser certaines caractéristiques matérielles (comme les encres et les écritures pour un manuscrit, le papier pour un manuscrit ou un imprimé).

Par delà les moyens techniques permettant d'accéder aux sources et la nécessité éventuelle de se déplacer physiquement pour les consulter, l'ampleur des difficultés rencontrées dépend de la nature de l'édition entreprise. Par exemple, et pour se limiter aux cas de publications présentées ici, on devine que si la publication bilingue de la seconde édition (1780) du Della moneta de Galiani a exigé une part d'enquête archivistique ${ }^{38}$, celle-ci est demeurée tout de même moins considérable que la recherche associée à la publication des Interwar Papers and Correspondence (1919-1939) de Harrod. Comme souvent, c'est en effet l'édition du versant actif de la correspondance d'un auteur qui impose le travail de prospection le plus conséquent. D. Besomi indique à cet égard (p. 232) que des lettres de Harrod ont été relevées dans près de 70 fonds - et l'on peut imaginer que la recherche effectuée s'est étendue à un nombre de dépôts bien plus grand, si l'on tient compte de ceux où rien n'a été trouvé.

Fréquemment, l'entreprise de prospection ne doit pas, en outre, se limiter à l'exploration des fonds des institutions publiques. D'une part, l'économiste éditeur, à l'instar de n'importe lequel de ses homologues œuvrant dans d'autres disciplines, doit suivre attentivement le marché des autographes, comme le font notamment P. Bridel et F. Mornati. Si ses moyens financiers ne lui permettent pas d'acheter certaines pièces, il doit demander au libraire ayant la responsabilité de la vente s'il est possible de les examiner avant que celle-ci ne se réalise ou bien, par la suite, le prier de contacter l'acquéreur afin de présenter une demande de consultation (ce genre de requêtes ne sont bien entendu pas forcément satisfaites). D'autre part, la recherche doit parfois s'étendre aux fonds détenus par des institutions ou des personnes privées, non seulement parce que certaines acquisitions résultent de ventes effectuées dans le passé, mais aussi parce qu'il arrive que les papiers de l'auteur qu'il s'agit de publier se trouvent, pour tout ou partie, en possession de ses

37. Le fonds des Archives personnelles et familiales (série AP) des Archives Nationales de France offre un bon exemple. L' "État sommaire des fonds" est téléchargeable en ligne (http://www.archivesnationales.culture.gouv.fr/chan/chan/pdf/ap/AP_etat_sommaire.pdf) mais les inventaires détaillés doivent être consultés sur place. Selon la recherche que l'on effectue, ces derniers catalogues ne sont parfois pas suffisamment précis. Le dépouillement du fonds s'impose alors. Cela peut nécessiter le dépôt d'une demande de consultation exceptionnelle qui n'est pas accordée immédiatement.

38. En premier lieu pour essayer de retrouver le manuscrit du texte. Rappelons que n'ont finalement été identifiés que des extraits de la traduction en français de Morellet ; l'un d'entre eux étant publié (pp. 63-64) au sein du présent numéro. 
descendants, ce qui peut imposer à l'éditeur un véritable travail de généalogiste. D. Besomi (pp. 231, 239-240) et F. Mornati (p. 216) relatent à ce propos les mésaventures qu'ils ont éprouvées avec plusieurs institutions privées, ces dernières limitant ou interdisant l'accès aux documents qu'elles détiennent et n'accordant pas le droit de les reproduire, sous quelque forme que ce soit. Fort de cette expérience, F. Mornati émet le souhait - dont on aimerait qu'il ne soit pas considéré comme un vœu pieux - que, via l'UNESCO, soient envisagés les moyens d'attribuer automatiquement "la propriété des papiers des savants du passé" à leurs "universités d'appartenance [...] avec, pour elles, l'obligation de les mettre facilement à la disposition des chercheurs qualifiés du monde entier" (ibid.).

\section{3. 2. Identifier l'auteur, authentifier le texte}

En tant que tel, le simple repérage de l'œuvre que l'on décidera de publier ne va pas forcément de soi. Il est parfois nécessaire, en effet, d'identifier les auteurs anonymes, de découvrir le nom de ceux qui se cachent derrière des pseudonymes, de distinguer la contribution de chacun lorsqu'on est confronté à un écrit rédigé à plusieurs mains ou encore de dévoiler les textes apocryphes. L'analyse du contenu du texte proprement dit, le rapprochement avec celui d'autres écrits ou la prise en compte du contexte historique doivent alors parfois s'accompagner d'un examen formel. Celui-ci peut consister dans l'étude des écritures manuscrites ou de la typographie, des filigranes et plus généralement du support matériel. Il peut aussi correspondre à un relevé et à une comparaison statistiques des termes employés dans le texte.

Ce type de problèmes se posent pour les œuvres économiques de la France de l'Ancien Régime ; époque où règne la censure ${ }^{39}$ et où la reconnaissance légale du statut et de la figure de l' "auteur" ne s'est pas encore imposée. Les éditeurs de Quesnay s'y sont heurtés, comme on pouvait naturellement s'y attendre. Ils nous rappellent (pp. 69, 73-74) à ce sujet que le chef de file des physiocrates ne s'est jamais affiché publiquement comme l'auteur de ses écrits ; lesquels furent rédigés, parfois en collaboration, sous le couvert de l'anonymat ou du pseudonymat. L'analyse des textes et de la correspondance les a ainsi conduits à confirmer ou à infirmer la rédaction par Quesnay d'une dizaine d'œuvres, tandis que l'étude des écritures sur les manuscrits de Mirabeau leur a permis de repérer les passages imputables à la main de Quesnay - le cliché d'un feuillet est à cet égard reproduit dans le présent numéro (p. 97), celui-ci faisant partie d'une note inédite de Quesnay, intégralement transcrite (pp. 92-96).

De telles difficultés d'identification et d'authentification interviennent aussi, bien sûr, pour les textes économiques publiés dans d'autres langues que le français

39. À propos de la censure et de l'anonymat des livres économiques, voir J.-C. Perrot (1984, pp. 301302) et C. Théré (1998, pp. 8-11). 
ou à d'autres époques. Comme l'indique C. Salvat (2000, p. 112, n. 28), il n'est toujours pas complètement acquis, à ce propos, que Galiani soit bel et bien l'unique auteur du Della moneta tandis que, par exemple, il a été montré par Gherity (1993) que Smith avait rédigé l'article sur la monnaie et les banques paru anonymement dans The Scots Magazine en décembre 1763. P. Groenewegen (1992, pp. 16-17) et surtout D. P. O'Brien et A. Darnell (1982) ont quant à eux multiplié les exemples d'identification relatifs à des textes économiques de la première moitié du XIX ${ }^{\mathrm{e}}$ siècle, rédigés en langue anglaise. D. E. Moggridge (1988c, pp. 80-81) a par ailleurs reconnu qu'il n'avait certainement pas pu repérer tous les textes anonymes et pseudonymes rédigés par Keynes, lors de la préparation des Collected Writings (1971-1989) de ce dernier. On évoquera enfin les difficultés que D. Besomi a rencontrées - et continue de rencontrer - dans son travail relatif à la publication de Prosperity and Depression (1937), pour faire le départ entre ce qui est l'œuvre de Haberler et ce qui émane d'autres personnes, notamment de certains économistes de la Société des nations.

\section{3. 3. Choisir le texte de base}

La question philologique du choix de texte de base se pose à n'importe quel éditeur, économiste ou non, dès qu'il existe plus d'une version de l'écrit qu'il envisage de publier. En général, le désir de respecter l'intention finale de l'auteur conduit à privilégier son dernier texte "revu et corrigé", à partir duquel seront signalées éventuellement les variantes associées à d'autres versions ${ }^{40}$, dans le cadre d'une publication dite variorum ${ }^{41}$.

Il en existe de nombreuses illustrations dans le domaine de l'édition de textes économiques. Ce principe a par exemple été adopté par A. S. Skinner lors de son édition de l'Inquiry into the Principles of Political Economy de J. Steuart, la version choisie ayant été "the corrected text of the 1767 edition" (A. S. Skinner 1966, p. v) réalisé par Steuart avant sa mort, en 1780. Il a été aussi suivi par Sraffa (1951, p. lx), ce dernier déclarant avoir accordé sa préférence à la troisième édition (1821) des Principles of Political Economy and Taxation (1817), c'est-à-dire "the last to be revised by Ricardo". De même, J. P. Potier et A. Tiran (pp. 159-160) indiquent qu'ils ont choisi comme ancres textuelles les deuxième (1840) et sixième (1841) éditions respectives du Cours complet d'économie politique pratique (18281829) et du Traité d'économie politique (1803) de J.-B. Say, "entièrement revue[s] par l'auteur". La septième et dernière édition (1871) des Principles of Political

40. Accompagnées de la mention de leur pagination (ou de leur foliotation) et, parfois, des manchettes, préface, avertissement, etc. de ces éditions.

41. Notons que le relevé préalable des variantes peut être aujourd'hui facilité par l'usage de moyens électroniques. Parmi ceux-ci figurent le logiciel MEDITE, initialement réservé aux études de génétique textuelle. J. Bourdaillet en avait fait une brève présentation lors du colloque "Éditer des textes économiques" (2006). Ce logiciel est téléchargeable gratuitement à l'adresse http://wwwpoleia.lip6.fr/ acasa/MEDITE/. 
Economy (1848) de J. S. Mill a été pareillement considérée comme son écrit de référence puisqu'elle est, affirme J. M. Robson (1965, p. 1xxx), "more reliable than any previous edition", compte tenu des "constant re-readings" de son auteur. La cinquième édition (1926), posthume, des Éléments d'économie politique pure (1874), a été également prise comme texte de base car, rappelle C. Mouchot (1992, p. 212), elle constitue "la forme la plus achevée" de l'œuvre de Walras. Dans une perspective toujours identique, la version française (1909) du Manuel d'économie politique (1905) ainsi que celle $(1917,1919)$ du Traité de sociologie générale (1916) de Pareto ont été choisies, explique F. Mornati (pp. 213-214), dans la mesure où elles comportaient des amendements par rapport à leur version initiale, en italien. Un autre exemple peut être donné avec les Collected Writings (1971-1989) de Keynes. D. E. Moggridge (1988c, p. 77) mentionne en effet que le document de base qui a été sélectionné pour tel ou tel écrit a été "the last corrected text that had passed through his hands"42.

Les arguments s'opposant à la primauté de la dernière version révisée d'une œuvre ne sont cependant pas négligeables. Ils sont, à notre connaissance, pas moins de six. Quatre d'entre eux conduisent à préférer la version initiale du texte ${ }^{43}$, tandis que les deux autres peuvent déboucher sur le choix de l'une de ses versions, et pas nécessairement de la dernière.

Le premier argument militant pour le choix de la première version du texte consiste à affirmer qu'il est de la sorte possible de saisir le surgissement créatif de l'œuvre, autrement dit ce qui est à proprement parler primordial dans celle-ci. Comme l'écrit G. Rudler (1923, p. 85) : "historiquement, la vraie date d'une œuvre est celle de son apparition. [...] C'est au moment de sa conception et de son exécution qu'une œuvre de pensée et d'art a son sens le plus exact. Ce qui suit, la série des retouches et des refontes [...] n'a de signification que par rapport à l'état premier du texte". Un deuxième argument part du constat suivant : il arrive que l'intention initiale de l'auteur finisse par disparaître au fil des révisions de son texte. Considérer que la dernière édition doit primer et que les versions antérieures - pour ne pas dire préliminaires - de l'œuvre doivent être réduites au rang de simples variantes serait, dans ce contexte, une erreur de perception téléologique: on prêterait à tort à l'auteur un mode d'écriture purement programmatique, donnant paradoxalement l'impression que la dernière version de son texte était déjà dissimulée au sein de la première $e^{44}$ et ne laissant donc aucune place à

42. À l'exception, néanmoins, de la plupart des lettres envoyées par Keynes. Les pièces privilégiées ont été en effet les copies carbones de ces lettres, principalement conservées à la bibliothèque du King's College de Cambridge, et non celles, parfois légèrement différentes, effectivement reçues par les destinataires.

43. À condition toutefois d'accorder une signification assez restrictive au terme de "texte". Dans le cas contraire, le fait de privilégier par exemple, en tant qu'écrit de référence, une phase rédactionnelle telle que l'esquisse d'un plan détaillé n'aurait en général pas de sens.

44. Une critique de cette conception erronée, qualifiée aussi de "rétrospective", est développée par A. 
l'improvisation. Troisièmement, la première édition est en principe celle qui subira le moins d'altérations "accidentelles" par rapport au texte original. Ces variantes, selon la terminologie du fameux bibliographe W. W. Greg (1950-1951), relèvent des graphies, de la mise en page, de la ponctuation, de la mise en caractères majuscules ou italiques, etc. Elles sont dues aux éditeurs successifs et non à l'auteur. Il peut arriver, enfin, que le texte le plus fidèle à la pensée de l'écrivain soit sa version initiale, lorsque celle-ci comporte des passages censurés par la suite.

Les deux raisons pouvant conduire à préférer telle ou telle version du texte, et pas nécessairement la dernière, ont été exposées par G. J. Stigler (1962, p. 285, n. 7). D'une part, au regard de l'histoire des idées, il importerait de prendre comme texte de base non pas l'écrit qui reflète l'intention finale de l'auteur, mais celui qui a eu le plus de retentissement et le plus d'influence sur les autres auteurs. D'autre part, la meilleure version d'une œuvre serait celle qui a été rédigée par l'auteur lorsqu'il était en pleine possession de ses facultés intellectuelles ${ }^{45}$. On devine que le choix d'une version particulière, associé à ces deux arguments, peut alors être quelque peu subjectif. Toujours est-il qu'il ne coïncide pas forcément avec le fait de considérer la dernière version du texte comme l'écrit de référence.

Il est possible de donner quelques illustrations de l'application de ces divers arguments - nous les limiterons au domaine de l'édition de textes économiques. A. Tiran et les autres responsables de la publication du Della moneta se sont ainsi écartés d'une vision téléologique en choisissant comme texte de base la première édition (1751), et non la seconde (1780) du texte de Galiani. Il en va de même de P. Taieb, qui a privilégié la première édition (1776) de la Richesse des nations, laquelle comporte de surcroît le moins d'altérations "accidentelles" par rapport au manuscrit original, comme l'indique W. B. Todd (1976, pp. 61-62) ${ }^{46}$. Le fait que la version initiale de l'œuvre puisse être la plus fidèle à la pensée de l'auteur est développé par les éditeurs de Quesnay. Ces derniers rappellent en effet (p. 72) qu'il existe des exemplaires non "cartonnés" de certains écrits du chef de file des physiocrates. Ceux-ci ne comportent pas, en d'autres termes, de pages réimprimées pour satisfaire la censure ${ }^{47}$. Et c'est peut-être aussi en partie compte tenu du poids de

Grésillon (1994, pp. 136-140). P. Ricœur (1989, pp. 216-217), dans sa présentation de la génétique textuelle, nous semble être victime de cette vision.

45. Cet argument sera notamment repris par J. M. Robson (1965, pp. lxxix-lxxx).

46. Bien qu'il ait quant à lui, avec les autres responsables de l'édition de Glasgow, accordé sa préférence à la troisième édition (1784) de la Richesse des nations car la première édition a été imprimée non à partir de l'autographe de Smith mais d'une copie réalisée par Gillies, tandis que la troisième édition a été relue et corrigée par Smith, reflétant ainsi sa volonté finale. Voir W. B. Todd (1976, pp. 61-63).

47. C'est du reste une des raisons pour lesquelles en dépit d'une même page de titre, des exemplaires d'un livre peuvent contenir un état du texte différent. Au sujet des modifications, en général, effectuées sous presse au XVIII ${ }^{\mathrm{e}}$ siècle, voir J. Rychner (1990, pp. 51, 63, 65-66) et W. B. Todd (1951-1952). Rappelons que les modifications en cours d'impression, quoiqu'elles ne soient pas explicitement mentionnées par les éditeurs économiques, interviennent encore aujourd'hui. 
la censure que l'un des manuscrits du Despotisme de la Chine (1767), qu'ils ont pris comme texte de référence, est de bien meilleure volée que sa version imprimée (pp. 72-73). En outre, estimer que l'édition choisie doit être celle qui a été écrite par l'auteur quand il était en pleine possession de ses facultés intellectuelles conduirait à sélectionner comme texte de base, affirme G. J. Stigler (1962, p. 285, n. 7), la troisième édition (1895) des Principles of Economics (1890) de Marshall plutôt que la huitième (1920). G. J. Stigler ajoute (ibid.) que si l'on considère que l'édition la plus influente d'un économiste est celle qui doit faire autorité, on doit pareillement exclure la dernière édition, posthume (1836), des Principles of Political Economy ${ }^{48}$ de Malthus au profit de la première (1820). En revanche, il conviendrait dans cette perspective de prendre comme écrit de référence, parmi les six éditions de l'Essay on the Principle of Population (1798) de Malthus, celui de la seconde (1803) édition ${ }^{49}$.

On doit cependant reconnaître que ces différents arguments conduisant à ne pas choisir la dernière version révisée d'un texte ont moins de prise chez les économistes que, notamment, chez les littéraires et les philosophes. Relatif aux altérations accidentelles qui augmentent au fil des éditions, le troisième d'entre eux est il est vrai moins pénétrant s'il s'agit de publier une poésie ou une pièce de théâtre - le respect de certaines graphies (c'est-à-dire de leur son) et de la ponctuation est alors souvent fondamental - que, par exemple, un texte d'économie mathématique ${ }^{50}$. L'essentiel, toutefois, ne nous semble pas résider en cela. Le fait est que ces arguments, de quelque nature qu'ils soient, nous paraissent simplement méconnus des économistes. Et, s'il existe bien entendu des cas de figure où le choix de la dernière édition approuvée par l'auteur s'impose naturellement, force est de reconnaître de façon plus générale que la signification même et l'importance qu'il convient d'accorder à son intention n'est débattue presque exclusivement que par les littéraires ou les philosophes ${ }^{51}$, précisément parce qu'elle ne relève pas de la théorie économique.

\footnotetext{
48. Le fait qu'on ne sache pas jusqu'à quel point Malthus est l'auteur de cette édition constitue un motif supplémentaire de son éviction, avancé par J. Pullen (1989), éditeur de la publication variorum des Principles of Political Economy de Malthus.

49. Comme le rappelle en effet P. James (1989, p. xiii), responsable de la publication variorum de ce texte : "it was the Essay of 1803 which made the greatest impact on contemporary thought, and the effect of some of the more famous passages, cancelled as early as 1806, extends even now [...] over discussions on demography and economics".

50. En dehors du cas, bien sûr, où ces altérations accidentelles introduisent un changement d'envergure ou carrément une erreur dans une démonstration ou un calcul.

51. Voir par exemple, et sans prétendre à l'exhaustivité, F. Bowers (1991), H. W. Gabler (1987), J. Guilhaumou (2006, notamment pp. 59-60, 65-68, 82-86), J. McGann (1991), J. McLaverty (1984), K. McLoughlin \& C. Gardner (2007), P. L. Shillingsburg (1982), Q. Skinner (1971-1972) et (2002), G. T. Tanselle (1976) et (1996).
} 


\section{3. 4. Établir les éléments de l'appareil critique}

Il est difficile d'assigner des limites au contenu de l'appareil critique, dans la mesure où les informations que l'on peut ajouter au texte n'ont pour bornes que l'imagination de l'éditeur, comme le rappelle M. J. Driscoll (2006, p. 261). Qu'il s'agisse de publier un économiste ou un auteur œuvrant dans une autre discipline, plusieurs éléments sont certes quasi incontournables : l'introduction de l'éditeur, les notes explicatives qui accompagnent le texte, l'index des noms (parfois enrichi d'indications biographiques), voire des thèmes.

D'autres éléments relevant de l'appareil critique peuvent être illustrés par certaines des publications présentées ici. Comme c'est le cas dans l'édition Quesnay, l'appareil critique peut ainsi être augmenté par un catalogue des manuscrits, une ou plusieurs chronologies, une biographie de l'auteur ou encore une bibliographie des références le concernant, ces dernières étant présentées de façon critique. On peut également y trouver des éléments aussi divers qu'un glossaire, comme dans l'édition (pp. 1xiii-lxix) du Della moneta de Galiani, et une description de la documentation dont l'auteur s'est servi pour élaborer sa propre œuvre, à l'instar de celle comprise dans l'édition Haberler (pp. 229-230, 232-233). L'appareil critique peut également accorder certains égards aux travaux éditoriaux antérieurs. Dans ce cas, il s'étendra à la mention des notes d'autres éditeurs, telles celles de H. Say sur la seconde édition (1840) du Cours complet d'économie politique pratique de J.-B. Say, ou encore les notes que W. Jaffé a jointes à sa traduction (1954) des Éléments d'économie politique pure de Walras. En outre, et ces deux dernières publications l'illustrent, un chapitre spécifique peut être réservé à l'histoire de l'édition en tant que telle.

L'appareil critique peut enfin comprendre un inventaire des documents allographes (feuillets de présentation des textes de l'auteur, papiers de ses proches concernant l'œuvre...), l'analyse du support matériel ayant servi de base à la publication - à savoir, s'il s'agit par exemple d'un manuscrit: type de papier, dimensions de celui-ci, espace graphique et instruments d'écriture.

\section{Seconde partie. Publier comment ?}

Que l'édition soit économique ou non, la question de sa forme revêt trois caractéristiques principales. La première renvoie au respect de la lettre du texte en tant que telle, c'est-à-dire à la fidélité qui doit être accordée à l'écrit original de l'auteur. L'éditeur est dans ce cas parfois confronté à l'enjeu suivant: parvenir à modifier la présentation formelle du texte, sans pour autant porter préjudice aux idées qu'il véhicule ou aux informations matérielles qu'il contient (2. 1.). La deuxième caractéristique concerne la nature des commentaires éditoriaux, par lesquels nous entendons la présentation générale du texte et les notes qui y sont associées. Jusqu'alors parfois représentés par de simples "avertissements" ou 
"préfaces", ceux-ci ne se sont véritablement répandus qu'à partir du début du XIX siècle, époque à laquelle a commencé de se développer l'édition scientifique ${ }^{52}$. Leur tonalité dominante était interprétative. Cette démarche éditoriale ne sera remise en cause qu'à la charnière des $\mathrm{XIX}^{\mathrm{e}}$ et $\mathrm{XX}^{\mathrm{e}}$ siècles, au profit d'une perspective informative qui s'est de nos jours très largement imposée (2. 2.). La troisième et dernière caractéristique associée à la forme de l'édition consiste dans son économie générale. Cet aspect renvoie essentiellement à l'ordre d'après lequel se présenteront les textes publiés ${ }^{53}$. Il s'agit ainsi d'opter pour une organisation chronologique ou thématique, à moins de choisir une solution intermédiaire entre ces deux types de présentation. Là aussi, des évolutions se sont fait jour, quoique de manière moins tranchée : les corpus de textes ont aujourd'hui tendance à être organisés sous un mode hybride, c'est-à-dire de façon "thématico-chronologique", tandis que ceux de correspondance sont très souvent présentés selon un principe chronologique (2. 3.).

\section{1. La question de la fidélité au texte original}

La question relative à la fidélité devant être accordée au texte original intervient lorsque l'intelligibilité de celui-ci ne va pas de soi, quand il s'agit de publier un brouillon manuscrit (2. 1. 1.) ou de traduire un texte (2. 1. 2.). Elle peut également se présenter lorsqu'on s'interroge sur la nécessité de rendre sa lecture plus fluide, en modernisant ses graphies, en amendant sa ponctuation, son accentuation etc. (2. 1.3.).

\section{1. Comment transcrire?}

Cette interrogation intervient quand on souhaite, en particulier, publier un manuscrit présentant des ratures et de façon générale plusieurs phases d'écriture. Deux principes de transcription peuvent alors être mobilisés : l'un consiste à respecter la topographie de la page ; l'autre à expliciter la succession temporelle des différentes strates d'écriture.

Prenons le cas de certains manuscrits de Sraffa, dont l'espace graphique est très complexe à appréhender - à telle enseigne que Sraffa lui-même, nous apprend H. Kurz (pp. 262-263), avait du mal à les lire a posteriori. Comment transcrire, par exemple, le feuillet (1956) du brouillon de Production of Commodities by Means of Commodities (1960) reproduit en couverture du présent numéro des Cahiers d'économie politique (ainsi que p. 277) ? On devine qu'une transcription respectant fidèlement l'espace graphique du feuillet serait absurde : cette transcription, dite

52. On trouvera quelques éléments à ce propos dans V. Tesnière (1985). Il n'existe cependant pas, à notre connaissance, d'étude particulière consacrée à l'histoire de l'édition scientifique.

53. Elle concerne aussi la question - que nous n'évoquerons pas ici - de l'emplacement des notes éditoriales et plus globalement de celui des autres éléments relevant de l'appareil critique. Cette question, dans le domaine de la littérature, a été envisagée par G. T. Tanselle (1972). 
diplomatique, n'apporterait rien, ou si peu, par rapport à une publication en facsimilé et, en l'espèce, il ne pourrait même pas être accordé à l'éditeur le mérite d'avoir déchiffré l'écriture de Sraffa, aisée à lire. La tâche de l'éditeur est, dans ce contexte, de tenter de distinguer les différentes phases d'écriture qui apparaissent entremêlées, c'est-à-dire de révéler comment, dans le temps, elles se sont superposées les unes aux autres. Cette transcription, dite diachronique, présente l'avantage d'offrir un texte lisible et d'en percevoir la maturation progressive. En outre, le confort de lecture qui découle de ce mode de transcription peut même se présenter lorsque le texte ne présente pas un grand nombre de ratures. Pour en rester à l'un des feuillets manuscrits publiés dans ce numéro, prenons un extrait (reproduit p. 64) de celui de la traduction par Morellet de la première édition du Della moneta (1751) de Galiani. Au bas de celui-ci, on lit "uses" suivi de "var poigued do sable". Transcrire ce passage par "une sachet plei[n] poignée de sable" ne faciliterait guère la lecture. Celle-ci serait en revanche plus aisée si l'on distinguait - et tel a été le choix d'A. Tiran (p. 63) - les deux états

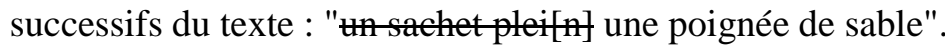

Par rapport à un mode de transcription diplomatique, le mode de transcription diachronique a cependant l'inconvénient, outre celui d'être plus coûteux en espace, de comporter potentiellement plus d'erreurs dans la mesure où il requiert un véritable effort de reconstruction de la part du transcripteur: des infidélités par rapport au véritable parcours génétique de l'œuvre peuvent dès lors se faire jour. Au demeurant, le choix de la transcription diplomatique semble parfois raisonnable. Tel est par exemple le cas, pour s'en tenir là encore à une des publications présentées et en partie reproduites ici (pp. 118-125), du mode de transcription de Sur les prix (1797-1801) de Bentham, tel que l'a établi et annoté Dumont : l'espace graphique des feuillets est suffisamment limpide pour être reproduit, comme l'indique N. Sigot, quasiment tel quel.

\section{1. 2. Comment traduire?}

En ce qui concerne la traduction ${ }^{54}$, un premier problème consiste à choisir entre une perspective "sourcière" et une perspective "cibliste" ${ }^{55}$. Comme lorsqu'il s'agit de traduire n'importe quel type d'écrits, il convient en effet d'opter entre le respect de la lettre originale du texte - quitte, s'il est par exemple question de traduire un texte anglais, à maintenir des termes dans cette langue ou à créer des anglicismes - et le sens qu'il contient - auquel cas on s'attachera à traduire le texte comme s'il avait été rédigé directement par l'auteur dans la langue de la traduction. Si le texte est relativement ancien, l'optique sourcière incitera à respecter le vocabulaire de

54. Nous nous contenterons ici d'un point de vue sommaire. Rappelons qu'un des ouvrages de référence sur la question de la traduction en général reste celui de G. Mounin (1963).

55. Nous reprenons la terminologie de J.-R. Ladmiral, dans A. Berman et J.-R. Ladmiral (1988). Sur ce point, voir aussi, par exemple, M. B. de Launay (1988). 
l'époque dans sa langue d'origine, tandis que l'optique cibliste tendra vers la modernisation dans la langue de la traduction. L'optique cibliste fournit un texte $a$ priori plus aisément compréhensible par le lecteur mais qui, en toute rigueur, devra être révisé au bout de quelques générations pour être précisément modernisé.

Si l'on s'en tient à quelques traductions en français, on notera par exemple que la perspective sourcière a été privilégiée dans la seconde édition (1999) de la traduction de Production of Commodities by Means of Commodities de Sraffa et celle (1995) de la Wealth of Nations de Smith. L'adaptation de ce dernier texte parue quelques années plus tard (2000-2005) reflète quant à elle une position intermédiaire. Cette option a aussi été suivie lors de la traduction (1992) des Principles of Political Economy and Taxation de Ricardo et dans celle du Della moneta de Galiani, à ceci près que cette dernière édition est bilingue : la possibilité d'une consultation aisée du texte italien amenuise les éventuels défauts de son adaptation en français.

\section{1. 3. Jusqu'à quel point faut-il respecter les graphies, la ponctuation, l'accentuation etc. du texte original ?}

Le problème de la fidélité à l'écrit original intervient en premier lieu lorsqu'on s'interroge sur la nécessité de moderniser les graphies ou de manière plus générale de corriger directement le texte ${ }^{56}$, notamment dans le cas où l'auteur rédige dans une langue dont il n'a pas une maîtrise parfaite. Le souci d'offrir le plus grand confort de lecture possible a par exemple conduit les éditeurs de Quesnay à restituer en français contemporain les graphies de ses textes imprimés ${ }^{57}$. Le parti pris de la modernisation, s'agissant d'un texte imprimé antérieur au milieu du XIX ${ }^{\mathrm{e}}$ siècle, peut aussi se justifier par le fait que la responsabilité des aspects formels du texte était alors, la plupart du temps, celle non de l'auteur mais de l'imprimeur, comme le signale B. Didier $\left(1996\right.$, p. 11) ${ }^{58}$. Il convient, enfin, d'avoir à l'esprit que la nature formelle d'un texte est a priori moins importante dans le domaine de l'économie que dans celui de la littérature ${ }^{59}$. Il est d'ailleurs difficile de citer aisément le nom d'économistes, du moins fameux, qui se sont assurés du bon déroulement, jusque dans ses moindres détails, du processus d'impression de leur œuvre, à l'exception

56. C'est-à-dire sans le signaler en usant par exemple de crochets droits. Bien entendu, ce genre de choix est parfois dicté par les contraintes financières (évoquées supra, pp. 17-18), quand elles imposent de faire paraître une édition "grand public".

57. Voir C. Théré, L. Charles et J.-C. Perrot (2005b, p. xxx).

58. Ces aspects formels doivent être rapprochés des variantes dites "accidentelles", déjà évoquées (supra, p. 18), entre le texte original de l'auteur et les diverses versions qui sortent de l'atelier de l'imprimeur.

59. Ce point est également mentionné supra, p. 25. 
peut-être de Keynes $^{60}$ et comme ont pu le faire en revanche, cela nous paraît plus connu, Sade $^{61}$, Balzac ${ }^{62}$, Sand ${ }^{63}$ ou d'autres écrivains ${ }^{64}$.

Cependant, il existe plusieurs arguments s'opposant à la modernisation ou à la correction de la nature formelle du texte, quel que soit le champ disciplinaire dans lequel il s'inscrit. Que l'on se propose de publier un imprimé ou un manuscrit, il ne faut pas surévaluer les difficultés de lecture associées à la conservation de la forme originale de sa lettre, y compris s'il s'agit d'un écrit relativement ancien. Par exemple, il nous semble vraiment qu'un lecteur francophone ne peut être, au pire, que légèrement incommodé par la lecture du débat entre Bodin et Malestroit dans sa langue du XVI siècle, tel que l'a publié J.-Y. Le Branchu (1934, pp. 49-177) en ne modifiant le texte des éditions princeps que de façon extrêmement modeste ${ }^{65}$. Le fait d'actualiser la forme d'un texte suppose en outre que celle-ci soit, par tous ses aspects, détachée de son fond et que l'auteur ne lui a réellement accordé aucune importance, ce qui ne va pas de soi. Troisièmement, et à l'instar d'une traduction axée sur l'optique cibliste, un texte modernisé devra nécessairement être révisé au bout de quelques générations. Enfin, il peut être considéré qu'un texte non modernisé présente un intérêt culturel et une saveur appréciables par tout honnête homme, qu'il soit économiste ou non.

S'il est question d'éditer plus particulièrement un manuscrit, ce respect pour la forme du texte s'impose encore plus fermement. En premier lieu parce que l'orthographe d'un auteur a pu évoluer dans le temps ; ce qui peut apporter des informations chronologiques lorsque ses écrits ne sont pas datés. Un tel cas peut intervenir, notamment, chez certains auteurs des Lumières, époque à laquelle l'orthographe n'était pas standardisée. Tandis que l'évolution des graphies paraît marginale chez Quesnay, elle est en tout cas patente, par exemple, chez un auteur tel que Condorcet : notre propre expérience d'éditeur de ce dernier nous en a convaincu $^{66}$. Si le fait de ne pas moderniser les graphies peut donc fournir des

60. Faisant le même constat que D. E. Moggridge (1988c, pp. 76-77), G. Dostaler (2005, pp. 323-324, n. 2) indique que "Keynes avait l'habitude de faire des corrections multiples et répétées sur les épreuves de ses livres. [...] [C]'est lui qui payait la production de ses ouvrages" : il pouvait donc disposer d'autant d'épreuves qu'il le désirait. Son souci de l' "esthétique" et ses grandes qualités littéraires (en particulier exposées par G. Dostaler (2002)) ont dû ainsi le conduire à être très pointilleux sur la bonne tenue, d'un point de vue formel, de l'impression de ses écrits.

61. À ce sujet, voir notamment B. Didier (1991, p. 202).

62. Consulter sur ce point S. Vachon (2000, passim).

63. Se reporter par exemple à sa Lettre à C. Edmond (août 1871, p. 92).

64. D. H. Reiman (1985, pp. 103-105, 115-120) cite à ce propos plusieurs auteurs britanniques des $\mathrm{XVIII}^{\mathrm{e}}$ et XIX ${ }^{\mathrm{e}}$ siècles. On doit reconnaître toutefois que certaines œuvres de ces derniers (par exemple Coleridge ou Godwin) n'étaient pas sans liens avec l'économie politique.

65. Comme le mentionne J. Y. Le Branchu (ibid., pp. 51, 71), seuls les "n" suscrits ont été supprimés ("mon̄oyes" donnant "monnoyes"), de même que les "u" doubles ("peuuent" donnant "peuvent").

66. Sur ce point, voir N. Rieucau (2009, pp. 42-43). Au sujet des éléments de datation ainsi fournis, voir ibid., pp. 46-49 et N. Rieucau (2004b, pp. 177-183). Quelles que soient les époques, rappelons que l'évolution de l'orthographe peut également être constatée lorsque l'auteur écrit dans une langue 
éléments de datation ${ }^{67}$, il peut aussi présenter à notre connaissance quatre autres avantages, que nous avons également éprouvés dans nos travaux sur Condorcet $(\mathrm{N}$. Rieucau 2004a). Premièrement, confirmer ou infirmer l'appartenance de certains manuscrits à un même projet intellectuel, que ces manuscrits soient ou non conservés dans un endroit identique. Deuxièmement, remarquer que la connaissance par le scripteur de tel ou tel auteur, parce qu'il en écrit le nom d'une façon que l'on juge fautive, relève ou non de sa culture orale ${ }^{68}$. Troisièmement, déceler dans le cadre d'une correspondance la plus ou moins grande familiarité qu'entretient l'auteur, surtout s'il ne rédige pas dans sa langue natale, avec le destinataire de sa lettre - parce qu'écrire à un proche impose en général une moindre attention formelle qu'écrire à un inconnu, à plus forte raison si ce dernier est un personnage éminent. Quatrièmement, distinguer certains copistes - dont les écritures quasi calligraphiées sont fort semblables - lorsque l'application ou les règles orthographiques et grammaticales dont ils ont coutume d'user sont différentes.

Ce respect de la forme du texte doit s'étendre jusqu'à la ponctuation, ou du moins celle-ci ne doit-elle être rétablie qu'a minima, comme ont choisi de le faire les éditeurs de Quesnay ${ }^{69}$. La ponctuation du XVIII ${ }^{\mathrm{e}}$ siècle demeure tout autant, si ce n'est davantage, axée sur l'articulation rythmique que sur l'articulation logique du discours et plusieurs de ses signes n'ont pas la même valeur que de nos jours ${ }^{70}$. Si l'on conçoit qu'elle puisse être parfois lacunaire dans un manuscrit et qu'il faut alors tenter d'y opérer quelques rétablissements, une entière modernisation peut se révéler dommageable, non seulement parce qu'elle priverait l'œuvre de son oralité et de son parfum originaux, mais aussi parce que, en cas d'erreur, elle pourrait en modifier le sens. On connaît à cet égard l'anecdote selon laquelle "Faute d'un point, Martin perdit son âne"...

\section{2. La nature des commentaires éditoriaux}

Dans le domaine de l'économie, les deux éditions (1802 et 1822) de la traduction de la Richesse des nations, par G. Garnier, constituent l'une des premières manifestations de l'apparition de commentaires éditoriaux un tant soit peu riches. Si la préface et les notes de ces deux éditions sont différentes, leur accent reste interprétatif. De même, les notes dites "explicatives et critiques" de J.-B. Say

qui n'est pas sa langue maternelle et dont il a fait un apprentissage progressif. Dans ce cas, la décision éventuelle de corriger directement, sans les mentionner, ses erreurs manifestes de plume doit être à notre sens exclue, toujours pour des raisons de datation.

67. Il est nécessaire, à cet égard, de respecter aussi les graphies des manuscrits dont on connaît la date d'écriture : leur comparaison avec celles de manuscrits non datés peut permettre d'attribuer une période de rédaction à ceux-ci.

68. Ce cas est fréquent pour les auteurs - en tout cas occidentaux - antérieurs au XIX $\mathrm{X}^{\mathrm{e}}$ siècle.

69. Voir C. Théré, L. Charles et J.-C. Perrot (2005b, p. xxxi).

70. Par exemple, les deux points expriment une pause dans l'énonciation de la phrase, celle-ci étant moins forte que le point mais plus forte que le point-virgule. Au sujet de la ponctuation au XVIII siècle, voir N. Catach (1996, pp. 35-42) et A. Lorenceau (1977). 
sur la traduction française (1819) de la première édition (1817) des Principles of Political Economy and Taxation de Ricardo sont en réalité plus "critiques" qu' "explicatives" ; à l'instar de celles de C. R. Prinsep qui interviennent sur la traduction anglaise (1821) de la quatrième édition (1819) du Traité de Say luimême, ou encore celles de C. C. Biddle sur ce texte. Des éditions d'une envergure plus vaste font montre, tout au long du siècle, d'une orientation similaire, que l'on songe à certains volumes de la Collection des principaux économistes (1841-1852) publiée chez Guillaumin ${ }^{71}$ ou à ceux de la Biblioteca dell'economista (1850-1868) édités par Ferrara.

À notre connaissance, une rupture avec cette démarche éditoriale n'interviendra qu'à la fin du XIX ${ }^{\mathrm{e}}$ siècle, lorsque seront publiées par Cannan, en 1896, les Lectures on Jurisprudence (alors parues sous le titre de Lectures on Justice, Police, Revenue and Arms) de $\mathrm{Smith}^{72}$. L'optique, informative, sera également celle de C. H. Hull et de F. B. Kaye lors de leur édition respective, en 1899 et 1904, des Economic Writings de Petty et de la Fable of the Bees $(1714,1723)$ de Mandeville. Sur le continent, G. Schelle suivra aussi cette démarche à l'occasion de la publication des Euvres de Turgot et documents le concernant (1913-1923), tout comme M. de Bernardi dans son édition des Écrits choisis et republiés (1933) de Dupuit. Cette même perspective sera préconisée, dans ce qui constitue sauf erreur de notre part le premier article exclusivement consacré aux questions éditoriales en matière d'économie politique, par L. Einaudi (1936). Une quinzaine d'années plus tard, ce dernier (1951, p. 158) considèrera à ce titre que le travail éditorial réalisé par Sraffa lors de la confection des Works and Correspondence de Ricardo est un modèle du genre, surpassant celui de Cannan.

L'optique informative est aujourd'hui largement partagée. Par exemple, R. D. Collisson Black (1988, p. 33), P. Bridel (1992b, p. 146), P. Groenewegen (1992, pp. 11-13), J. Rojahn (2000, pp. 409-410), G. de Vivo (2009, p. 99) et, dans le présent recueil, L. Charles et C. Théré (pp. 80-81), P. Dockès et C. Mouchot (p. 200-201), D. Besomi (pp. 226-228) ou encore H. Kurz (pp. 271-273) s'en font explicitement les avocats. En fin de compte, la seule forme de publicité accordée de nos jours aux éditions interprétatives consiste dans leur diffusion en fac-similé, évoquée précédemment (supra, p. 9), que ce soit sous format papier ou électronique.

71. Celle-ci publie notamment, en les augmentant de nouvelles notes critiques, les traductions de Smith ("revue et corrigée" : 1843, vol. 5-6) et de Ricardo (1847, vol. 13) que nous venons de citer.

72. Cannan explique ainsi dans son "Introduction" (1896, p. xxi) : "The notes are purely explanatory and historical. They are intended to help the reader to understand the text, to judge of the accuracy of the report, and to compare it with the authorities open to Adam Smith and with the subsequent development of his thought in the Wealth of Nations. The most conscientious effort has been made to resist the temptation to which commentators of the Wealth of Nations have generally succumbed, of using the text as a mere clothesline on which to hang editorial opinions on economic theory". Cannan adoptera la même démarche à l'occasion de son édition, en 1904, de la Whealth of Nations. 
Le reproche essentiel fait aux éditions interprétatives est leur nature assez rapidement obsolète - l'analyse que fera l'éditeur de la pensée de l'auteur sera forcément daté $^{73}$ - et subjective. Il s'agit donc, à l'inverse, d'être le moins intrusif possible en se limitant à des commentaires de nature factuelle, c'est-à-dire précisant la nature du texte, en indiquant les écrits ou les personnages auxquels l'auteur fait allusion, en donnant des éléments sur le contexte qui accompagne la rédaction de son œuvre, en éclairant le sens de certains termes ou de certains passages, en mentionnant les variantes, etc. S'effacer derrière l'auteur et se contenter de mettre son texte à la disposition du public de la façon la plus neutre qui soit est, en d'autres termes, l'objectif poursuivi. Cela ne signifie pas, évidemment, que l'éditeur puisse systématiquement s'abstraire de qualités interprétatives, lorsqu'il s'agit, justement, de déterminer quel est le point de vue de l'auteur, ou encore de déchiffrer son écriture, de rapprocher tel ou tel de ses propos avec un autre passage de son œuvre ou d'associer une pièce manuscrite à une phase rédactionnelle précise. Mais il demeure que la dimension interprétative est alors mobilisée comme un moyen au service de la dimension informative, et non comme une fin en soi.

En somme, et sur ce point précis relevant du "comment éditer", la nature des commentaires éditoriaux n'est plus aujourd'hui affaire de choix, du moins voit-on mal ce qui pourrait en être les motivations, tant les raisons militant en faveur de l'optique informative paraissent raisonnables.

\section{3. L'organisation générale du corpus publié}

Comme le suggère G. Busino (1992, pp. 379-380), lorsqu'il relate les débats qui intervinrent lors de la préparation de l'édition Pareto, la perspective chronologique permet de retracer la genèse et l'évolution de la pensée de l'auteur, tandis que la perspective thématique peut notamment viser à respecter la répartition d'un certain nombre de champs disciplinaires (soit, pour Pareto, la sociologie, l'économie et les sciences politiques) ou bibliographiques (par écrits principaux ou types d'écrits).

À chacun de ces modes de présentation sont fréquemment associées des difficultés intrinsèques. Le souhait d'établir un ordre chronologique entre divers écrits, qu'ils soient économiques ou non, peut être contrarié par la non-datation de ceux-ci par l'auteur (par exemple de ses lettres ou de ses textes manuscrits) ou l'impossibilité d'attribuer une date unique ou précise à tel ou tel écrit (quand on distingue la date de rédaction de cet écrit de celle de sa publication, lorsque sa rédaction ou sa publication se sont étalées dans le temps, ou encore quand celui-ci a connu un certain nombre d'éditions ou de traductions différentes). La perspective

73. De même, faire référence aux analyses qui ont succédé à celles de l'auteur publié est à éviter. Cette démarche rétrospective est en effet figée temporellement. Elle sera par conséquent nécessairement dépassée à terme. Sur ce point, D. Besomi (2006) a très clairement répondu à W. Young (2005). 
thématique, quant à elle, conduit fréquemment à des découpages arbitraires (lorsqu'il s'agit par exemple de choisir entre la classification des disciplines privilégiée par l'auteur et celle qui prévaut aujourd'hui, ou encore quand un texte relève de plusieurs disciplines à la fois ou qu'une lettre, rangée dans la correspondance, s'inscrit dans un champ disciplinaire constituant par ailleurs l'un des volets de l'édition - à moins bien sûr de la publier à deux reprises).

Il n'existe donc pas d'organisation générale idéale et celle-ci doit fréquemment être déterminée au cas par cas. Les éditeurs de Walras indiquent ainsi (p. 200) qu'ils ont opté pour une présentation thématique pour ne pas livrer de manière totalement démembrée les Études d'économie sociale et les Études d'Economie politique appliquée - celles-ci, parues respectivement en 1896 et 1898, sont en effet constituées par des articles "publiés sur de longues périodes" (ibid.). Cela étant, les éditions chronologiques semblent aujourd'hui peu courantes. Pour autant que nous le sachions, la dernière édition chronologique volumineuse d'un économiste d'envergure sont les Euvres de Turgot et documents le concernant, publiées par G. Schelle entre 1913 et $1923^{74}$. L'orientation délibérément historique défendue par Schelle (1913, pp. 5-6) et les notices bio-bibliographiques situées en ouverture de chaque volume paraissent, dans le cas présent, avoir dicté une telle organisation. Il est assez difficile de donner une explication à cette relative rareté des éditions chronologiques, lesquelles sont également peu fréquentes en dehors de l'économie ${ }^{75}$. On imagine cependant que l'entreprise éditoriale devenant de plus en plus souvent un travail collectif, la division d'une œuvre en plusieurs séries thématiques - à l'intérieur desquelles la dimension chronologique pourrait néanmoins être conservée - permet de confier la préparation des textes de chacune d'entre elles à une personne précise, spécialiste de tel ou tel domaine de la pensée de l'auteur. Outre cette raison pratique, il est possible que les exigences accrues de la critique savante, coïncidant avec le souci d'établir des éditions didactiques, aient rendu plus naturelle l'idée de présenter l'œuvre de l'auteur selon un certain nombre de catégories. Une telle organisation a été, par exemple, privilégiée dans les Collected Works (1963-1991) de J. S. Mill, ceux (1988-) de Hayek, les Collected Writings (1971-1989) de Keynes, la Marx-Engels-Gesamtausgabe (1975-), les Euvres de Charles Gide (1997-) ou, parmi les publications commentées dans le présent volume, les éditions de Quesnay et de Harrod.

Inversement, les éditions de lettres, qu'elles soient ou non comprises dans un corpus de plus vaste envergure, semblent se présenter de plus en plus rarement "par

74. Nous excluons par conséquent ici les publications de moindre ampleur, telle que les Jeremy Bentham's Economic Writings (1952-1954), ceux-ci ne comprenant qu'une quinzaine de textes réunis dans trois volumes. Quoique nettement plus imposant, le corpus des Works of Irving Fisher (1997) doit être aussi écarté car les trois derniers de ses quatorze volumes marquent une rupture avec l'ordre chronologique.

75. Parmi les éditions en cours de réalisation, on peut citer, en particulier, les Nietzsche Werke (1967-), les Writings of Charles S. Peirce (1982-) et les CEuvres complètes (2002-) de Zola. 
correspondants". Ce type de présentation était fréquent dans les éditions, d'économistes ou non, du $\mathrm{XIX}^{\mathrm{e}}$ siècle. Sans doute était-ce parce que la correspondance active d'un auteur n'était pas encore systématiquement considérée comme faisant partie intégrante de son œuvre publiable, tandis que sa correspondance passive était plus encore négligée, sauf si elle était le fait de quelque sommité. Dans ce contexte, les lettres n'étaient éditées, après avoir été sélectionnées, que par "correspondants illustres", rubriques parfois enrichies d'une "correspondance générale". Depuis la seconde moitié du $\mathrm{XX}^{\mathrm{e}}$ siècle, l'exigence d'exhaustivité paraît coïncider avec la prédominance d'une organisation chronologique, comme l'illustrent par exemple les éditions de référence de la correspondance d'Overstone, Jevons et Marshall. La seule entorse à ce mode de présentation intervient lorsqu'une ou plusieurs lettres sont identifiées a posteriori, tandis que l'édition dans son ensemble n'est pas encore achevée. On peut citer à cet égard les correspondances de Smith, Ricardo, ou encore L. Walras - la publication de la correspondance de Pareto, quant à elle, constitue un cas extrême, puisque les six volumes de lettres ont été édités, nous rappelle F. Mornati (p. 215), à mesure de la découverte de celles-ci.

\section{Conclusion}

À la lumière des considérations précédentes, il apparaît que de la détermination du contenu de l'œuvre jusqu'à son organisation générale, en passant par la question du choix du texte de base, du mode de transcription des manuscrits ou encore des éléments de l'appareil critique, le travail éditorial en histoire de la pensée économique ne comporte pas de réelles particularités par rapport à celui qui intervient dans d'autres domaines de la connaissance. Si spécificités il y a a ${ }^{76}$ du moins, celles-ci ne sauraient assurément pas motiver la négligence dont il se trouve affecté $^{7}$. Cette négligence est, évidemment, d'autant plus dommageable qu'elle touche la diffusion - et la qualité - de la littérature primaire qui gouverne les orientations de notre discipline et sans laquelle, plus fondamentalement, celle-ci n'aurait pas de raison d'être ${ }^{78}$.

76. La principale d'entres elles nous semble relever de la nécessité moins systématique, en comparaison d'une ouvre littéraire, de respecter certains aspects formels du texte. À ce sujet, voir supra, pp. 25, 29.

77. Cette négligence, mentionnée au début de notre étude (supra, p. 9), paraît notamment bien plus grande que chez les philosophes, non seulement parce que les compétences habituelles de ces derniers sont parfois proches de celles des littéraires, mais aussi parce que certaines questions posées par les matières éditoriales - en particulier celle (évoquée supra, p. 25) du sens et de l'importance qu'il s'agit de donner à "l'intention" de l'auteur - constituent de véritables interrogations philosophiques.

78. C'est ainsi que P. Dockès et C. Mouchot (p. 203) n'hésitent pas à affirmer que la publication des Cuvres Économiques Complètes des Walras à "contribué à revivifier" l'histoire de la pensée économique en France, tout en contribuant à une meilleure et plus ample connaissance de la pensée du Maître de Lausanne et de celle de son père. Un " 'revival parétien' ", déclare G. Busino (1989, p. xi), a pareillement vu le jour suite à la parution des CEuvres Complètes du successeur de L. Walras à la Chaire d'Économie Politique de l'Université de Lausanne. En se limitant aux éditions présentées ici, la 
L'acte éditorial requiert en outre des qualités interprétatives dont l'historien de la pensée économique use dans son analyse des textes, sans vraiment songer au profit qu'il pourrait en retirer s'il s'intéressait justement à la publication des auteurs qu'il commente. Nous avons en effet indiqué qu'il était fréquemment impossible, sans mobiliser de réelles capacités analytiques, de dévoiler l'identité d'un auteur dissimulée par l'anonymat, de dater tel ou tel de ses textes, de les situer dans l'économie générale de son œuvre et du contexte intellectuel de son temps, de déchiffrer son écriture manuscrite, de déceler l'objectif qu'il s'assigne, de déterminer les écrits qui feront partie du corpus publié, d'élaborer la structure de celui-ci, d'établir son index thématique, etc.

À ce titre, il convient de rappeler que des économistes de renom - Say, Marx, Jevons, Walras, Keynes, Sraffa... - ont exercé des responsabilités éditoriales ${ }^{79}$. Pour défendre une discipline dont on sait qu'elle est en perte de vitesse, les historiens de la pensée économique se plaisent parfois à signaler que de grands économistes furent souvent de grands historiens; ils devraient également se souvenir que certains d'entre eux furent aussi de grands éditeurs.

réévaluation et la meilleure compréhension de la pensée de Galiani (p. 60), Sismondi (passim), Say (pp. 151, 159, 164), Bentham (pp. 102-103, 106-111) ou encore Juglar (pp. 175, 177, 182, 184-188) sont ouvertement avancées par leurs éditeurs.

79. Comme leur intitulé l'indique, Editing Economists and Economists as Editors, les actes publiés par P. Bridel (1992a) étaient précisément centrés sur cet objet. 


\section{Appendice : la solution électronique}

Nous avons évoqué (supra, pp. 19, 22 (n. 41)) le fait que l'entreprise de prospection, de reproduction et de collation des textes, constituant le début du travail éditorial, était aujourd'hui facilitée par l'usage de l'outil informatique. Comme nous allons à présent l'expliquer, le recours à ce même outil permet de résoudre aussi un certain nombre de problèmes directement associés au choix du contenu et de la forme de l'édition. Cela étant, nous verrons que le support électronique n'est pas exempt de tout inconvénient en comparaison du support papier.

Plus l'édition que l'on envisage de publier sur format papier est riche, notamment par la présence d'un grand nombre de notes éditoriales ${ }^{80}$ ou auctoriales ${ }^{81}$, plus sa lecture risque d'être inconfortable. Il est certes possible de rendre cette lecture plus aisée grâce à un système de renvois, un jeu sur la mise en page, la création d'encadrés, de marges différentes, la variation des polices de caractères, de leur dimension, etc ${ }^{82}$. Il demeure cependant que la capacité d'accueil offerte par le support papier est physiquement limitée et qu'une contradiction existe, en l'espèce, entre le souci d'exhaustivité et celui de lisibilité. L'un des atouts de l'édition électronique consiste dans le fait de pouvoir, en grande partie, surmonter cette contradiction. En effet, le multi-fenêtrage et les liens hypertextuels véhiculent un potentiel synoptique plus important et plus souple que le livre imprimé, tandis que l'encre électronique promet à terme un confort de lecture du texte, en tant que tel, équivalent à celui-cis ${ }^{83}$.

Par là même, des problèmes de présentation plus spécifiques peuvent être résolus. Il en va ainsi de la publication sur papier des marginalia, c'est-à-dire des notes manuscrites qu'un auteur rédige en marge d'exemplaires, manuscrits ou imprimés, en sa possession. Pour des raisons matérielles, certains éditeurs sont contraints de ne mentionner, outre bien sûr le contenu de la note, que sa localisation

80. Que ces notes consistent en des commentaires ou en la mention de variantes entre différentes versions du texte.

81. Ce second type de notes se rencontre d'ailleurs aujourd'hui plus fréquemment dans des écrits économiques, historiques ou philosophiques que dans des écrits littéraires. Sur l'histoire de la note de bas de page, voir A. Grafton (1997).

82. De nature synoptique, le dispositif mis en place à l'occasion des publications variorum des Éléments d'économie politique pure de Walras puis du Traité d'économie politique de J. B. Say est à ce titre exemplaire.

83. Cela ne signifie pas que l'outil informatique puisse promettre des éditions exhaustives et fidèles au sens strict. Même le plus parfait des fac-similés - reproduit en l'occurrence sur format numérique - ne pourra restituer totalement les traces de la manipulation du papier, la marque et la contremarque de son filigrane, le reflet des éléments chromatiques, les caractéristiques de la reliure du livre, l'éventuelle dorure sur sa tranche, etc. ; en somme l'ensemble des caractéristiques matérielles aujourd'hui considérées comme susceptibles d'être porteuses de sens ou, pour reprendre l'expression des éditeurs de Quesnay (2005a, p. xix), "scientifiquement loquace[s]". 
et non l'extrait auquel elle se rapporte. C'est de cette façon que se présentent les notes de J.-B. Say en marge de la Richesse des nations de Smith et des Definitions in Political Economy (1827) de Malthus, telles qu'elles ont été respectivement publiées par H. Hashimoto (1980) et H. Kitami \& K. Mizuta (2002). Dans le cadre de l'édition des Euvres complètes de Say, J. P. Potier et A. Tiran indiquent (p. 159) que les notes en question seront en revanche accompagnées de l'extrait concerné. Ce procédé, déjà adopté par Sraffa lors de sa publication des Notes on Malthus's Principles of Political Economy (1820) de Ricardo, sera aussi repris, signale H. Kurz (p. 269), lors de l'édition des marginalia de Sraffa lui-même ${ }^{84}$. En toute rigueur, on devine toutefois que la publication de ce genre d'inscriptions devrait comprendre la totalité de l'écrit annoté, non seulement parce que plusieurs d'entre elles se rapportent à une grande étendue de texte, mais également pour percevoir que tel ou tel passage n'a justement pas été commenté. Certains éditeurs, comme ceux de Quesnay, ont renoncé face à un tel impératif, relativement lourd d'un point de vue matérie ${ }^{85}$. Compte tenu de sa capacité d'accueil bien plus grande, une édition électronique peut en revanche contenir l'intégralité de l'écrit annoté en le reproduisant en format texte ou en fac-similée ${ }^{86}$.

Une autre illustration des atouts de l'électronique en matière de capacité d'accueil consiste dans le traitement des états antérieurs du texte, plus précisément s'il s'agit d'adopter un mode de transcription diachronique. Ce procédé de transcription présente l'inconvénient, nous l'avons indiqué (supra, p. 28), d'être plus exigeant en espace que le procédé de transcription diplomatique et de comporter, parfois, une part d'arbitraire lors de la reconstruction des états successifs du texte. Dans le cadre d'une édition électronique, le premier de ces problèmes ne se pose plus, tandis qu'on peut imaginer qu'une publication du manuscrit en fac-similé, accompagnant sa transcription sous un mode diachronique, laisse ouverte la possibilité pour l'utilisateur chevronné de la corriger. Sauf erreur de notre part, les économistes éditeurs n'ont pas encore œuvré dans ce domaine, à la différence de leurs homologues philosophes ou littéraires ${ }^{87}$.

84. Nous ne savons pas encore, en revanche, si cette publication des marginalia sera accompagnée de la mention intégrale des marques "muettes" que constituent les traits tirés par Sraffa en marge de ses lectures - tels que ceux, évoqués par H. Kurz (p. 272), relatifs à un passage d'Il materialismo storico e la filosofia di Benedetto Croce (1948) de Gramsci. Notons que les éditeurs des E Euvres complètes (1968-) de Voltaire ont été jusqu'à mentionner ce genre de traces, ainsi que les angles de pages cornées par le Patriarche de Ferney.

85. Voir C. Théré, L. Charles, J.-C. Perrot (2005, p. xxvi).

86. Dans ce dernier cas, il arrive cependant que les droits de reproduction soient plus difficiles à obtenir que pour une édition papier, comme le rappelle le Committee on Scholarly Editions (2006, p. 26) et, dans le présent numéro, D. Besomi (p. 239-240).

87. Sous la responsabilité de P. d'Iorio, le cas de l' "Hypernietzsche" (http://www.hypernietzsche.org/base.html) nous semble constituer un bon exemple. Pour un développement relatif aux possibilités de l'électronique en matière de représentation des états successifs du texte, voir J. L. Lebrave (2007). Ajoutons que l'électronique facilite également la représentation du texte selon un mode de transcription diplomatique accompagné de fac-similés. Une 
Le risque d'arbitraire associé à une édition papier, dont nous venons de donner un exemple, peut également être réduit, grâce à l'usage de l'édition électronique, sous d'autres points de vue. La subjectivité véhiculée par le choix de tel ou tel mot pour construire l'index des matières est ici absente puisque l'utilisateur peut user directement de la recherche "plein-texte". De plus, grâce à sa capacité d'accueil, une version électronique peut dissiper le problème de la sélectivité, et donc de la part d'arbitraire des choix éditoriaux, qui s'impose parfois à un ouvrage sur format papier. D. Besomi, s'il rappelle (p. 236-238) ces deux éléments, indique aussi (p. 237) que le support électronique atténue dans une certaine mesure les difficultés résultant du choix du texte de base puisque l'intégralité des variantes peuvent en principe être mentionnée (à moins que les diverses versions du texte ne soient simultanément disponibles). On devine aussi, de manière plus large, que les problèmes liés à l'organisation générale de l'édition se posent de façon moins aiguë car la présentation formelle de celle-ci peut-être aisément modifiée. Un cas exemplaire est fourni par la correspondance. À l'instar des lettres échangées par L. Euler ${ }^{88}$, A. M. Ampère ${ }^{89}$, Keller ${ }^{90}$ ou H. Poincaré $^{91}$, celles relatives à Harrod, sur le site que D. Besomi lui a dédié ${ }^{92}$, peuvent ainsi être consultées de façon chronologique ou bien par correspondants.

Un dernier avantage, et non des moindres, présenté par le support électronique si on le compare au support papier réside dans son caractère constamment amendable, à condition bien entendu qu'il s'agisse d'une version en ligne et non sur cd-rom. Il est alors possible d'augmenter régulièrement le nombre de textes disponibles ou de corriger tel ou tel aspect de l'édition déjà existante. Pour en rester au domaine de l'édition économique, on peut par exemple renvoyer au site réalisé par P. Taieb ${ }^{93}$ (fréquemment mis à jour par l'addition de nouveaux textes, principalement du XVIII ${ }^{\mathrm{e}}$ siècle), ou de nouveau à celui consacré à Harrod, réalisé par D. Besomi ${ }^{94}$.

Pour autant, l'édition électronique n'est pas une panacée, comme le souligne également D. Besomi (p. 237-240). Tout d'abord parce qu'en réduisant la part de subjectivité qui peut grever une publication sur format papier, le support numérique ôte paradoxalement d'autant la valeur qui peut être accordée au travail éditorial. L'utilisateur risque alors de crouler sous les informations $\mathrm{s}^{95}$. Du moins devra-t-il lui-

illustration, d'ailleurs assez fameuse, est fournie par l'édition électronique des Manuscrits de Madame Bovary (http://bovary.univ-rouen.fr/).

88. Euler's Correspondence : http://math.dartmouth.edu/ euler/.

89. Correspondance d'Ampère : http://www.ampere.cnrs.fr/correspondance/.

90. Gottfried Keller Briefwechsel : http://www.gottfriedkeller.ch/.

91. La correspondance d'Henri Poincaré : http://www.univ-nancy2.fr/poincare/chp/.

92. Ce site est présenté pp. 236-240, 244-252.

93. Le jardin aux sentiers qui bifurquent : http://www.taieb.net/.

94. L'actualisation et la correction possible de ce site sont évoquées p. 237.

95. Ce point est aussi souligné par G. T. Tanselle, dans l'avant-propos du recueil Electronic Textual 
même prendre en charge plusieurs des tâches assumées par l'éditeur d'une version papier, lesquelles pourront consister dans la sélection des écrits à la source de son commentaire, le choix du texte de base ou encore la détermination de certains motsclefs, compte tenu de l'absence d'index. De ce point de vue, l'utilisateur peut à son tour prendre des décisions discutables, ce d'autant plus qu'il est a priori, face aux choix en question, moins compétent qu'un éditeur. L'autre inconvénient majeur de l'édition électronique résulte de sa viabilité bien moins grande que celle de l'édition papier. Finalement, si son contenu peut être mis à jour presque sans inconvénient, ce ne peut être qu'à court terme, c'est-à-dire à l'horizon d'une quinzaine d'années. Au-delà, la technologie et les techniques dont elle est le fruit seront dépassées.

Aussi doit-on conclure, en suivant là encore D. Besomi (p. 239), que les qualités de l'édition électronique sont nuancées par rapport à l'édition papier. Tant et si bien qu'elle doit être pour le moment considérée comme son complément, non pas son substitut.

Editing, édité par L. Burnard et alii (2006) : "In an electronic edition, where there is unlimited space, all the relevant texts are likely to be presented in full, and readers may feel disoriented without some direction from the editor", G. T. Tanselle (2006, p. 5). 


\section{RÉFÉRENCES}

\section{Sources imprimées (et manuscrites)}

Note : Les articles compris dans le présent numéro des Cahiers d'économie politique et répertoriés dans son sommaire ne sont pas référencés ici.

Allen Woody (1972), "Les listes Metterling", Pour en finir une bonne fois pour toutes avec la culture, trad. : M. Lebrun, Opus 2, Points, 1973, pp. 7-15.

Aristote, L'Éthique à Nicomaque, trad. : R.-A. Gauthier \& J.-Y. Jolif, Louvain-laNeuve, Paris, Sterling (Virginie), Peeters, 2002, 4 vol.

Aristote, Les politiques, Paris, Flammarion, 1993.

Aspromourgos Tony (2000), "Historical Scholarship and Publication, or - Why Do Commercial Publishers Exist?", History of Economics Review, Summer, n³2, pp. 1-9.

Baker William \& Womack Kenneth (2000), Twentieth Century Bibliography and Textual Criticism: An Annotated Bibliography, Westport, Greenwood.

Baudelaire Charles (1861-1863), "Carnet", Euvres complètes, Paris, Gallimard, Bibliothèque de la Pléiade, 1983, t. I, pp. 710-780.

Bentham Jeremy (1787), Défense de l'usure ou Lettres sur les inconvénients des lois, qui pèsent sur le taux de l'intérêt de l'argent, suivi d'un mémoire sur les prêts d'argent, par Turgot, trad. : S.-A. Bazard, Paris, Malher \& Cie, 1828.

Bentham Jeremy (1787-1795), Jeremy Bentham's Economic Writings, W. Stark (éd.), London, George Allen \& Unwin, 1952-1954, 3 vol.

Bentham Jeremy, Euvres économiques, N. Sigot (éd.), (à paraître).

Bentham Jeremy (1797-1801), Sur les prix, Bibliothèque de Genève, Ms. Dumont 50.

Bentham Jeremy (1802), Esquisse d'un ouvrage en faveur des pauvres adressée à l'éditeur des Annales d'agriculture, trad. : A. Duquesnoy, Paris, Imprimerie des Sourds-Muets, an X.

Bentham Jeremy (1811), "[Manuel d'économie politique]", Théorie des peines et des récompenses, $2^{\text {nde }}$ éd. (1818), rédigée en françois d'après les manuscrits, par M. É. Dumont, t. II, vol. 2, pp. 293-439 : Livre IV ("Des Encouragemens par rapport à l'Industrie et au Commerce"), Paris, Londres, Bossange \& Masson.

Béraud Alain (1992), "Introduction à la Quatrième partie: Les économies classiques", dans A. Béraud \& G. Faccarello (dir.), Nouvelle histoire de la pensée économique, t. 1 : Des scolastiques aux classiques, Paris, La Découverte, pp. 305-308.

Berman Antoine \& Ladmiral Jean-René (1988), "Les sourciers et les ciblistes Entretien", Préfaces, nº 7 pp. 80-82 
Bernardi Bruno (2002), "Introduction" de J. J. Rousseau (1755, 1758), Discours sur l'économie politique, Paris, Vrin, pp. 7-36.

Bertrand Aliénor (1998), Logique naturelle et principes de l'action humaine dans l'œuvre de Condillac, thèse pour le doctorat en philosophie, Université Paris XNanterre.

Besomi Daniele (2006), "A note on Textual editing: A Rejoinder to Young", Journal of the History of Economic Thought, vol. 28, n 3, sept., pp. 375-381.

Biblioteca dell'economista - Première et seconde série (1850-1868), F. Ferrara (éd.), Torino.

Biziou Michaël (2003), Adam Smith et l'origine du libéralisme, Paris, PUF.

Bowers Fredson (1991), "Authorial Intention and Editorial Problems", Text, 5, pp. 49-61.

Bridel Pascal (éd.) (1992a), "Editing Economists and Economists as Editors", Revue européenne des sciences sociales, $\mathrm{t} . \mathrm{XXX}, \mathrm{n}^{\circ} 92$.

Bridel Pascal (1992b), "The Lausanne Lectures in Pure Economics: From Walras to Pareto (how and what to publish, if anything at all!)", dans P. Bridel (éd.) (1992a), pp. 145-169.

Burnard Lou \& alii (éd.) (2006), Electronic Textual Editing, New York, Modern Language Association of America.

Busino Giovanni (1989), "Note liminaire", dans V. Pareto, "Lettres et correspondances - Compléments et additions", Euvres complètes, G. Busino (dir.), Genève, Droz, t. XXX, pp. vii-xxi.

Busino Giovanni (1992), "Examen de conscience d'un éditeur déçu et néanmoins convaincu de la nécessiter [sic] de bien éditer les œuvres fondamentales en sciences sociales", dans P. Bridel (éd.) (1992a), pp. 379-387.

Cannan Edwin (1896), "Editor's Introduction", dans A. Smith (1763 / 1896), pp. xixxxiv.

Catach Nina (1996), La ponctuation, Collec. Que-sais-je ? (n² 2818), Paris, PUF.

Charles Loïc, Perrot Jean-Claude, Théré Christine (2009), " 'Anglo-Saxon vs Continental Schorlarship: On Critical Editions of Economic Classics': A Comment on de Vivo", Contributions to Political Economy, vol. 28, pp. 93-98.

Chartier Roger \& Martin Henri-Jean (dir.) (1983-1986), Histoire de l'édition française, Paris, Fayard-Cercle de la Librairie, 1989-1990, 4 t.

Child Josiah (1754), Traités sur le commerce suivis [trad. : V. de Gournay] des Remarques de Jacques Vincent de Gournay, S. Meyssonnier (éd.), "Collection" des Cahiers d'économie politique, Paris, L'Harmattan, 2008.

Collection des principaux économistes (1841-1852), Paris, Guillaumin, 15 vol.

Collison Black Robert Denis (1988), "Editing the Papers of W. S. Jevons", dans D. E. Moggridge (1988a) (dir.), pp. 19-42.

Committee on Scholarly Editions (2006), " "Guidelines for Editors of Scholarly Editions", Annotated Bibliography: Key Works in the Theory of Textual Editing ", dans L. Burnard \& alii (éd.) (2006), Electronic Textual Editing, Modern Language Association of America, pp. 23-46. 
Condillac Étienne Bonnot de (1754), Traité des sensations [suivi du] Traité des animaux, Paris, Fayard, 1984.

Condillac Étienne Bonnot de (1755), Traité des animaux, dans E. B. de Condillac (1754), pp. 309-429.

Condillac Étienne Bonnot de (1776), Le commerce et le gouvernement, Paris, Genève, Slatkine, 1980.

Condorcet Jean-Antoine-Nicolas Caritat de (1772-1794), Tableau historique des progrès de l'esprit humain - Projets, Esquisse, Fragments et Notes, P. Crépel \& J. P. Schandeler (dir.), Paris, Ined, 2004.

Deleule Didier (1979), Hume et la naissance du libéralisme économique, Paris, Aubier Montaigne.

Didier Béatrice (1991), "Conclusions", La Fin de l'Ancien Régime - Sade, Rétif, Beaumarchais, Laclos : Manuscrits de la Révolution I, Manuscrits Modernes, Presses Universitaires de Vincennes, pp. 197-203.

Didier Béatrice (1996), "Présentation", dans B. Didier \& J. Neefs (dir.), "Éditer des manuscrits - Archives, Complétude, Lisibilité", Manuscrits modernes, Presses Universitaires de Vincennes, pp. 5-20.

Dockès Pierre \& alii (1990), "Introduction générale", dans A. \& L. Walras, Euvres Économiques Complètes, vol. I : A. Walras : Richesse, liberté et société, Paris, Economica, pp. ix-xlvii.

Dockès Pierre \& Servet Jean-Michel (1992), "Les lecteurs de l'armée morte - Note sur les méthodes en histoire de la pensée économique", dans P. Bridel (éd.) (1992a), pp. 341-364.

Dostaler Gilles (2002), "Discours et stratégies de persuasion chez Keynes", Sciences de la Société, n55, pp. 122-136.

Dostaler Gilles (2005), Keynes et ses combats, Paris, Albin Michel.

Driscoll Matthew James (2006), "Levels of Transcription", dans L. Burnard \& alii (éd.) (2006), pp. 254-261.

Dulac Georges \& Karp Sergueï (2007), "Guides des archives", Les Archives de l'Est et la France des Lumières, Ferney-Voltaire, Centre international d'étude du XVIII ${ }^{\mathrm{e}}$ siècle, vol. 1.

Dupuit Jules (1934) [= 1933], De L'utilité et de sa mesure - Écrits choisis et republiés, M. de Bernardi (éd.), Torino, La Riforma Sociale, Paris, Marcel Giard.

Einaudi Luigi (1936), "Come non si devono ristampare i nostri classici", dans L. Einaudi (1953, pp. 40-47).

Einaudi Luigi (1951), "Dalla leggenda al monumento", dans L. Einaudi (1953, pp. 155-161).

Einaudi Luigi (1953), Saggi bibliografici e storici intorno alle dottrine economiche, Roma, Edizioni di storia e letteratura.

Eltis Walter (1995), "L'abbé de Condillac and the Physiocrats", History of Political Economy, 27 (2), pp. 217-36.

Fisher Irving, The Works of Irving Fisher, W. J. Barber (éd.), London, Pickering \& Chatto, 1997, 14 vol. 
Foucault Michel (1969), "Qu'est-ce qu'un auteur ?", Dits et écrits - 1954-1988, t. I : 1954-1969, Gallimard, 1994, pp. 789-812.

Fusco Antonio-Maria (1992), "Éditions anastatiques, éditions diplomatiques, éditions critiques : lumières et ombres d'une tâche ingrate", dans P. Bridel (éd.) (1992a), pp. 325-339.

Gabler Hans Walter (1987), "The Text as Process and the Problem of Intentionality", Text, 3, pp. 107-116.

Galiani Ferdinando (1750) [= 1751], Della Moneta - Libri cinque, Napoli, G. Raimondi ; $2^{\mathrm{e}}$ éd. (1780), Napoli, nella stamp. Simoniana.

Galiani Ferdinando (1780), De la monnaie - Della moneta, éd. et trad. sous la dir. d'A. Tiran, Paris, Economica, 2005.

Galilée (1598-1634), "Ricordi autografi", Le Opere di Galileo Galilei, Firenze, Tipografia Barbèra, vol. XIX, 1907, pp. 130-206.

Gherity James Arthur (1993), "An Early Publication by Adam Smith", History of Political Economy, vol. 25, n², pp. 241-282.

Gide Charles, Les Euvres de Charles Gide, M. Pénin (éd. gén.), Paris, L'Harmattan, 1997-.

Grafton Anthony (1997), The Footnote: A Curious History, Cambridge (Mass.), Harvard University Press.

Gramsci Antonio (1948), Il materialismo storico e la filosofia di Benedetto Croce, Milano, Einaudi.

Greg Walter Wilson (1950-1951), "The Rationale of Copy-Text", Studies in Bibliography, 3, pp. 19-36.

Grésillon Almuth (1994), Éléments de critique génétique - Lire les manuscrits modernes, Paris, PUF.

Groenewegen Peter (1992), "Editing the classics in the Antipodes with special reference to the problem of identifying anonymous authors", dans P. Bridel (éd.) (1992a), pp. 9-24.

Guilhaumou Jacques (2006), Discours et évènement. L'histoire langagière des concepts, Besançon, Presses universitaires de Franche-Comté.

Haberler Gottfried von (1937), Prosperity and Depression. A Theoretical Analysis of Cyclical Movements, $1^{\text {ère }}$ éd., Genève, Société des Nations.

Haberler Gottfried von (1937), Prosperity and Depression. A Theoretical Analysis of Cyclical Movements, D. Besomi (éd.) (à paraître).

Harrod Roy Forbes (1919-1939), The Interwar Papers and Correspondence of Roy Harrod, D. Besomi (éd.), Cheltenham, Elgar, 2003.

Hashimoto Hitoshi (1980), "Notes inédites de J.-B. Say qui couvrent les marges de la Richesse des nations et qui la critiquent : rédigées avec une introduction", Kyoto Sangyo University - Economic and Business Review, vol. 7, pp. 53-81.

Hayek Friedrich August, The Collected Works of Friedrich August Hayek, W. W. Bartley \& S. Kresge (éd.), London, Routledge,1988-.

Hecht Jacqueline (1989), "De la révolution scientifique à la révolution culturelle : l'enseignement de l'économie politique", dans J.-M. Servet (dir.), Idées 
économiques sous la Révolution (1789-1794), Presses Universitaire de Lyon, pp. 35-80.

Hume David (1739), "Les passions", Traité de la nature humaine, Livre II, trad. : J.-P. Cléro, Paris, GF-Flammarion, 1991.

[Hume David] (anonyme) $(1741,1742)$, Essays, Moral \& Political, $1^{\text {ère }} \& 2^{\mathrm{e}}$ éd., dans D. Hume, Essais moraux, politiques et littéraires, éd. Définitive (1777), trad. : J.-P. Jackson, Paris, Alive, 1999, pp. 33-303.

Hume David (1752, 1760), Discours politiques, trad. : F. Grandjean, Mauvezin, Trans-Europ-Repress, 1993.

Hume David (1754-1762), The History of England from the Invasion of Julius Caesar to the Revolution in 1688, réimpr. de l'éd. de 1778 : LibertyClassics, 1983, 6 vol.

James Patricia (1989), "Editor's Introduction", dans T. R. Malthus (1803), pp. ix-xv.

Jevons Stanley (1850-1882), "Correspondence", Papers and correspondence of William Stanley Jevons, vol. 2-5 (1973-1987), R. D. Collison Black (éd.), London, Macmillan.

Jolink Albert (1992), " 'No man Is An Island' The Case of Jan Tinbergen", dans P. Bridel (éd.) (1992), pp. 261-277.

Juglar Clément, Écrits économiques, M. Dal-Pont Legrand \& alii, (éd.) (à paraitre).

Keynes John Maynard, The Collected Writings of John Maynard Keynes, D. E. Moggridge \& E. Johnson (éd.), London, MacMillan, 1971-1989.

Kitami Hiroshi \& Mizuta Ken (2002), "Les notes de J.-B. Say sur l'édition originale de 'Definitions in Political Economy' de T. R. Malthus", Bulletin of the Center for Historical Social Science Literature, Hitotsubaschi University, vol. 22, mars, pp. 2-7.

Ladmiral Jean-René \& Berman Antoine (1988), "Les sourciers et les ciblistes Entretien avec Jean-René Ladmiral et Antoine Berman", Préfaces, n ${ }^{\circ}$, pp. 8082.

Lapidus André (1995), "Introduction à une Histoire de la pensée économique qui ne verra jamais le jour", Les Cahiers Charles Gide, n ${ }^{\circ}$, pp. 85-114.

Launay Marc Buhot (de) (1988), "Théories de la traduction", Préfaces, nº ${ }^{\circ}$, pp. 8284.

Laurent Évelyne \& Marco Luc (1996), "Le Journal des économistes ou l'apologie du libéralisme (1841-1940)", dans L. Marco (dir.), Les revues d'économie en France (1751-1994), Paris, L'Harmattan, pp. 79-120.

Le Branchu Jean-Yves (1934), Écrits notables sur la monnaie, XVI siècle, de Copernic à Davanzati, Paris, Alcan, t. I.

Lebrave Jean-Louis (2007), "Du visible au lisible : comment représenter la genèse ?", Genesis, n 27, pp. 11-17.

Levan-Lemesle Lucette (dir.) (1986), Les problèmes de l'institutionnalisation de l'économie politique en France au XIX ${ }^{e}$ siècle, Économies et Sociétés - Série P. $E$., Presses Universitaires de Grenoble, $n^{\circ} 6$.

Lorenceau Annette (1977), "La ponctuation au XVIII ${ }^{\mathrm{e}}$ siècle - L'effort de systématisation des grammairiens-philosophes", dans N. Catach (dir.), La 
ponctuation - Recherches historiques et actuelles, Paris, Besançon, CNRSHESO (dactylographie), pp. 127-149.

McGann Jerome John (1991), "What is Critical Editing?", Text, 5, pp. 15-30.

McLaverty James (1984), "The Concept of Authorial Intention in Textual Criticism", The Library, $6^{\text {th }}$ ser., 6 (June), pp. 121-138.

McLoughlin Kate \& Gardner Carl (2007), "When is Authorial Intention not Authorial Intention?", European Journal of English Studies, vol. 11, n ${ }^{\circ}$, Apr., pp. 93-105.

Malthus Thomas Robert (1803), An Essay on the Principle of Population, $2^{\mathrm{e}}$ éd. [variorum], P. James (éd.), Cambridge, Cambridge University Press, 1989, 2 vol.

Malthus Thomas Robert (1820), Principles of Political Economy, Considered with $a$ View to their Practical Application, $1^{\text {st }}$ éd., London, J. Murray; $2^{\text {nd }}$ edition, with considerable additions from the author's own manuscript and original memoir, London, W. Pickering, 1836.

Malthus Thomas Robert (1827), Definitions in Political Economy, New York, A. M. Kelley, 1986.

Mandeville Bernard de (1714, 1723), The Fable of the Bees: or Private Vices, Publick Benefits, F. B. Kaye (éd.), Oxford, Clarendon Press, 1924, 2 vol.

Marchal Jean (1938), "L'école psychologique et la théorie de la valeur", Mélanges dédiés à M. le professeur Henry Truchy, Paris, Librairie du Recueil Sirey, pp. 300-348.

Marshall Alfred (1868-1924), The Correspondence of Alfred Marshall, J. K. Whitaker (éd.), Cambridge, Cambridge University Press, 1996, 3 vol.

Marshall Alfred (1890), Principles of Economics, $3^{\mathrm{e}}$ éd. (1895), London, Macmillan; $8^{\mathrm{e}}$ éd. (1920), London, Macmillan.

Marx Karl, Engels Friedrich, Marx-Engels-Gesamtausgabe [dite "MEGA 2"], Berlin, Karl Dietz Verlag (éd. : 1975-1989), Akademie Verlag (éd. : 1989-).

Mill John Stuart, The Collected Works of John Stuart Mill, J. M. Robson (éd.), Toronto, University of Toronto Press, London, Routledge and Kegan Paul, 1963-1991, 33 vol.

Mill John Stuart (1848), "The Principles of Political Economy with Some of Their Applications to Social Philosophy", $7^{\mathrm{e}}$ éd. (1871), J. M. Robson (éd.), The Collected Works of John Stuart Mill, 1965, vol. II-III.

Mirabeau Victor Riqueti (marquis de) \& Quesnay François (1757-1759), Traité de la monarchie, G. Longhitano (éd.), "Collection" des Cahiers d'économie politique, Paris, L'Harmattan, 1999.

Mirabeau Victor Riqueti (marquis de) \& Quesnay François (1757-1760), "Bref état des moyens pour la restauration de l'autorité du roi et de ses finances", G. Weulersse (éd.), Revue d'histoire économique et sociale, 1913, vol. VI, pp. $177-211$.

Moggridge Donald Edward (éd.) (1988a), Editing Modern Economists, XXII ${ }^{\text {nd }}$ Annual Conference on Editorial Problems, 7-8 nov. 1986, New York, AMS Press. 
Moggridge Donald Edward (1988b), "Introduction", dans D. E. Moggridge (1988a) (éd.), pp. 11-17.

Moggridge Donald Edward (1988c), "On Editing Keynes", dans D. E. Moggridge (1988a), pp. 67-90.

Moggridge Donald Edward (1992), "Editing: - What might Economist-Editors learn from others?", dans P. Bridel (éd.) (1992a), pp. 365-378.

Morand Émile (1912), La théorie psychologique de la valeur jusqu'en 1776, thèse pour le doctorat de l'université, Bordeaux, Imprimerie de l'Université.

Morellet André (abbé) (fin des années 1750 ?), Des principes de la valeur, tiré de Galiani della moneta, Bibliothèque Municipale de Lyon, MS 2526b, f. 1-18.

Mouchot Claude (1992), "L'édition synoptique des Éléments d'économie politique pure", dans P. Bridel (éd.) (1992a), pp. 209-231.

Mounin Georges (1963), Les problèmes théoriques de la traduction, Gallimard.

Nietzsche Friedrich, Nietzsche Werke: kritische Gesamtausgabe, G. Colli \& M. Montinari (éd.), Berlin, New York, W. de Gruyter, 1967-.

Nietzsche Friedrich, Euvres philosophiques complètes, Gallimard, 1967-1990, 13 vol.

O'Brien Denis Patrick \& Darnell Adrian (1982), Authorship Puzzles in the History of Economics - A statistical Approach, London, MacMillan Press ltd.

Orain Arnaud (2003), "Decline and Progress: The Economic Agent in Condillac's Theory of History", European Journal of the History of Economic Thought, 10 (3), pp. 379-407.

Orain Arnaud (2004), Choix individuels, Morale et Théorie de la valeur dans l'œuvre de l'abbé de Condillac (1714-1780), thèse pour le doctorat en sciences économiques, Université Paris I-Panthéon Sorbonne.

Overstone Samuel Jones Lloyd (1804-1883), The Correspondence of Lord Overstone, D. P. O'Brien (éd.), London, Cambridge University Press, 1971, 3 vol.

Pareto Vilfredo, CEuvres complètes, G. Busino (éd. [gén.]), Genève, Paris, Droz, 1964-.

Pareto Vilfredo (1906) [= 1905], "Manuel d'économie politique", trad. : A. Bonnet, 1909, Euvres complètes, vol. vII, 1966.

Pareto Vilfredo (1916), "Traité de sociologie générale", trad. : P. Boven, 19171919, Euvres complètes, vol. XII, 1968.

Peirce Charles Sanders, Writings of Charles S. Peirce: A Chronological Edition, M. H. Fisch (éd.), Bloomington, Indiana University Press, 1982-.

Perrot Jean-Claude (1984), "Nouveautés : l'économie politique et ses livres", dans R. Chartier \& H.-J. Martin (dir.) (1983-1986), t. II, pp. 298-326.

Petty William, The Economic Writings of Sir William Petty, together with The Observations upon the Bills of Mortality, more probably by Captain John Graunt, C. H. Hull (éd.), Cambridge, The University Press, 1899, 2 vol.

Pullen John (1989), "Editorial Procedures in this Variorum Edition", dans T. R. Malthus, Principles of Political Economy, J. Pullen (éd.), Cambridge, Cambridge University Press, pp. xi-xiii. 
Quesnay François, François Quesnay et la physiocratie, Paris, Ined, 1958, 2 t.

Quesnay François, Euvres économiques complètes et autres textes, C. Théré, L. Charles \& J.-C. Perrot (éd.), Paris, Ined, 2005, 2 t.

Quesnay François (1760), "Tableau économique avec ses explications", Euvres économiques complètes et autres textes, t. I, pp. 439-526.

Quesnay François (1767), "Despotisme de la Chine", Hagley Museum (Wilmington, E. U.), Fonds Du Pont de Nemours, Euvres économiques complètes et autres textes, t. II, pp. 1005-1114.

Reiman Donald Henry (1988), "Gentlemen Authors and Professional Writers: Notes on the History of Editing Texts of the $18^{\text {th }}$ and $19^{\text {th }}$ Centuries", dans R. Landon (éd.), Editing and Editors, $\mathrm{XXI}^{\text {st }}$ Annual Conference on Editorial Problems, 1-2 nov. 1985, New York, AMS Press, pp. 99-136.

Ricardo David, The Works and Correspondence of David Ricardo, P. Sraffa \& M.H. Dobb (éd.), Cambridge, Cambridge University Press, 1951-1973, 11 vol.

Ricardo David, Euvres complètes, trad. : P. [i. e. F.] Constancio \& A. Fonteyraud, augmentées de notes de J.-B. Say, de nouvelles notes et de commentaires par Malthus, Sismondi, MM. Rossi, Blanqui, etc., et précédées d'une notice... par A. Fonteyraud, Collection des principaux économistes, Paris, Guillaumin, 1847, vol. 13.

Ricardo David, "Additional Letters", The Works and Correspondence of David Ricardo, vol. 11 (1973), pp. ix-xxx.

Ricardo David, "Letters", The Works and Correspondence of David Ricardo, vol. 69 (1952-1955).

Ricardo David (1810-1811), "Notes on Bentham 'Sur les prix' ", The Works and Correspondence of David Ricardo, vol. 3 (1951), pp. 259-341.

Ricardo David (1817), Des principes de l'économie politique et de l'impôt, trad. (de la $3^{\mathrm{e}}$ éd. : 1821) : C. Soudan et alii, Paris, Flammarion, 1992.

Ricardo David (1817), Principes de l'économie politique et de l'impôt, traduction de

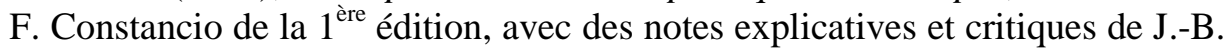
Say, Paris, J.-P. Aillaud, 1819, 2 t.

Ricardo David (1817), "On the Principles of Political Economy and Taxation", $3^{\mathrm{e}}$ éd. (1821), The Works and Correspondence of David Ricardo, vol. 1 (1951).

Ricardo David (1820), "Notes on Malthus's Principles of Political Economy", The Works and Correspondence of David Ricardo, vol. 2 (1951).

Ricœur Paul (1989), "Regards sur l'écriture", dans L. Hay (dir.), La naissance du texte, José Corti, pp. 213-220.

Rieucau Nicolas (2004a), "Établissement du texte" (resp.) de J.-A.-N. de Condorcet (1772-1794).

Rieucau Nicolas (2004b), "Présentation des textes isolés", dans J.-A.-N. de Condorcet (1772-1794), pp. 177-183

Rieucau Nicolas (2009), L'économie d'un savant: l'œuvre de Condorcet, des archives à l'interprétation, Synthèse pour l'habilitation à diriger des recherches, Université Paris 8.

Robson John Mercel (1965), "Textual Introduction", dans J. S. Mill, The Collected 
Works, vol. II: Principles of Political Economy with some of Their Applications to Social Philosophy (books I-II), J. M. Robson (éd.), University of Toronto Press, Routledge \& Kegan Paul, pp. 1xv-lxxxvii.

Rojahn Jürgen (2000), "Publishing Marx and Engels after 1989: The Fate of the Mega", Nature, Society and Thought, vol. 13, n4, pp. 407-420.

Rousseau Jean-Jacques, CEuvres complètes, G. Gagnebin \& M. Raymond (éd.), Paris, Gallimard, Bibliothèque de La Pléiade, 1959-1995, 5 t.

Rousseau Jean-Jacques (1750), "Discours sur les sciences et les arts", Euvres complètes, t. III (1964, réimpr. : 1991), pp. 1-30.

Rousseau Jean-Jacques (1755), "Discours sur l'origine et les fondements de l'inégalité", Euvres complètes, t. III (1964, réimpr. : 1991), pp. 109-223.

Rousseau Jean-Jacques $(1755,1758)$, "Discours sur l'économie politique", Euvres complètes, t. III (1964, réimpr. : 1991), pp. 239-278.

Rousseau Jean-Jacques (1760-1769), "Projet de Constitution pour la Corse", Cuvres complètes, t. III (1964, réimpr. : 1991), pp. 899-950.

Rousseau Jean-Jacques (1761), "Julie, ou La Nouvelle Héloïse", J. J. Rousseau. Euvres complètes, t. II (1961, réimpr. : 1990), pp. 1-794.

Rousseau Jean-Jacques (1770), "Les Confessions", J.-J. Rousseau. CEuvres complètes, t. I (1959, réimpr. : 2001), pp. 1-656.

Rousseau Jean-Jacques (1771), "Considérations sur le gouvernement de Pologne", Cuvres complètes, t. III (1964, réimpr. : 1991), pp. 951-1041.

Rudler Gustave (1923), Les techniques de la critique et de l'histoire littéraire, Paris, Genève, Slatkine Reprints, 1979.

Rychner Jacques (1984), "Le travail de l'atelier", dans R. Chartier \& H.-J. Martin (dir.) (1983-1986), t. II, pp. 46-70.

Salvat Christophe (2000), "Morellet et la diffusion de la pensée économique italienne", Il Pensiero economico italiano, 8, pp. 99-124.

Sand George (août 1871), "Lettre à Charles Edmond", Impressions et souvenirs, Paris, Librairie nouvelle, pp. 92-93.

Say Jean-Baptiste, Euvres complètes, E. Blanc \& alii (éd.), Paris, Economica, 2003-, 10 vol.

Say Jean-Baptiste (1803), Traité d'économie politique ou Simple exposition de la manière dont se forment, se distribuent et se consomment les richesses, $6^{\mathrm{e}}$ éd. entièrement revue par l'auteur, Paris, Guillaumin, 1841.

Say Jean-Baptiste (1803), Traité d'économie politique ou Simple exposition de la manière dont se forment, se distribuent et se consomment les richesses, $6^{\mathrm{e}}$ éd. (1841), dans J.-B. Say, Euvres complètes, E. Blanc \& alii (éd.), Paris, Economica, vol. I* et I **, 2006.

Say Jean-Baptiste (1821), A Treatise on Political Economy; or The Production, Distribution, and Consumption of Wealth. Translated from the fourth edition [(1819)] of the French by C. R. Prinsep, with notes by the translator. London, Longman, Hurst, Rees, Orme \& Brown, 2 t.

Say Jean-Baptiste (1821), A Treatise on Political Economy; or The Production, Distribution, and Consumption of Wealth. Translated from the fourth edition 
[(1819)] of the French by C. R. Prinsep, with notes by the translator. Translation of the introduction [Discours préliminaire], and additional notes by Clement C. Biddle, Boston, Wells and Lilly, 2 t.

Say Jean-Baptiste (1828-1829), Cours complet d'économie politique pratique, $2^{\mathrm{de}}$ éd. entièrement revue par l'auteur, Paris, Guillaumin, 1840.

Schelle Gustave (1913), "Turgot - Sa vie et ses œuvres - Turgot étudiant et magistrat (jusqu'en 1761)", Euvres de Turgot et documents le concernant, t. I (1913), pp. 1-76.

Schumpeter Joseph Alois (1954), Histoire de l'analyse économique, trad. : J.-C. Casanova, Gallimard, 1983, 3 t.

Schumpeter Joseph Alois (1970-posth.), Théorie de la monnaie et de la banque, C. Jaeger \& O. Lakomski-Laguerre (éd. et trad.), "Collection" des Cahiers d'économie politique, Paris, Budapest, Torino, L'Harmattan, 2005, 2 vol.

Sgard Jean \& Volpilhac-Auger Catherine (dir.) (1999), "La Notion d'œuvres complètes", Studies on Voltaire and the Eighteenth Century, ${ }^{\circ} 370$, pp. 1-12.

Shillingsburg Peter Leroy (1982), "Key Issues in Editorial Theory", Analytical \& Enumerative Bibliography, vol. 6, $\mathrm{n}^{\circ}$ 1, pp. 3-16.

Silberner Edmund (1939), "Un manuscrit inédit de David Ricardo sur le problème monétaire", Revue d'histoire économique et sociale, vol. XXV, pp. 195-259.

[Sismondi] Jean-Charles-Léonard Simonde (de), Euvres économiques complètes, P. Bridel (dir.) (à paraître).

Skinner Andrew Stewart (1966), "Preface", dans J. Steuart (1767, 1780), pp. v-vii.

Skinner Quentin (1971-1972), "Motives, Intentions and the Interpretation of Texts", New Literary History, 3, pp. 393-408.

Skinner Quentin (2002), Visions of Politics, vol. 1: "Regarding Method", Cambridge University Press.

Smith Adam, The Glasgow Edition of the Works and Correspondence of Adam Smith, Oxford, Clarendon Press, 1976-1978, 6 vol.

Smith Adam, "Correspondence of Adam Smith", The Glasgow Edition of the Works and Correspondence of Adam Smith, vol. 6, 1977.

Smith Adam (1759), "The Theory of Moral Sentiments", D. D. Raphael \& A. L. MacFie (éd.), 6 éd. (1790), The Glasgow Edition of the Works and Correspondence of Adam Smith, vol. 1, 1976.

Smith Adam (1762-1763, 1766), "Lectures on Jurisprudence", R. L. Meek, D. D. Raphael, P. G. Stein (éd.), The Glasgow Edition of the Works and Correspondence of Adam Smith, vol. 5, 1978.

Smith Adam (1763), Lectures on Justice, Police, Revenue and Arms delivered in the University of Glasgow, E. Cannan (éd.), Oxford, Clarendon Press, 1896.

Smith Adam (1763), "Thoughts Concerning Banks, and the Paper-Currency of Scotland", dans J. A. Gherity (1993, pp. 274-278).

Smith Adam (1776), Recherches sur la Nature et les Causes de la Richesse des Nations, traduction nouvelle [de la $5^{\mathrm{e}}$ éd.] avec des notes et observations par Germain Garnier, $1^{\text {ère } e ́ d . ~: ~ P a r i s, ~ A g a s s e, ~ 1802, ~} 5$ vol. ; $2^{\mathrm{e}}$ éd. : Paris, Agasse, 1822,6 vol. 
Smith Adam (1776), Recherches sur la nature et les causes de la richesse des nations ; trad. : G. Garnier entièrement revue et corrigée ; et précédé d'une notice biographique par M. Blanqui avec les commentaires de Buchanan, G. Garnier, Mac Culloch... [et al.] ; augmentée de notes inédites de J.-B. Say ; et d'éclaircissements historiques par M. Blanqui ; Collection des principaux économistes, Paris, Guillaumin, 1843, vol. 5-6.

Smith Adam (1776), An Inquiry into the Nature and Causes of Wealth of Nations (1776), $5^{\mathrm{e} e ́ d . ~(1789): ~ E . ~ C a n n a n ~(e ́ d .), ~ L ~}$

ondon, Methuen \& co., 1904, 2 vol.

Smith Adam (1776), "An Inquiry into the Nature and Causes of the Wealth of Nations", $3^{\text {e }}$ éd. (1784), R. H. Campbell, A. S. Skinner \& W. B. Todd (éd.), The Glasgow Edition of the Works and Correspondence of Adam Smith, vol. 2a et $2 b, 1976$.

Smith Adam (1776), Enquête sur la nature et les causes de la richesse des nations ; trad. (de la $1^{\text {ère }}$ éd. : 1776) : P. Taieb, Paris, PUF, 1995, 5 vol.

Smith Adam (1776), Recherche sur la Nature et les Causes de la Richesse des Nations; trad. (de la $3^{\mathrm{e}}$ éd. : 1784) : J.-M. Servet (dir.), Paris, Economica, 2000-2005, 3 vol.

Sraffa Piero (1951), "Introduction", The Works and Correspondence of David Ricardo, vol. I, p. xiii-lxiv.

Sraffa Piero (1956), [Manuscrit de] Production of Commodities by Means of Commodities, Cambridge, Trinity College Library, D3/12/1-115.

Sraffa Piero (1960), Production of Commodities by Means of Commodities, trad. : S. Latouche : Production de marchandises par des marchandises, $2^{\mathrm{e}}$ éd. révisée et présentée par G. Deleplace, Paris, Dunod, 1999.

Sraffa Piero, Unpublished Papers and Correspondence, P. Garegnani \& H. Kurz (éd. gén.) Cambridge, Cambridge University Press, 3 vol. (à paraître).

Steuart James (1767), An Inquiry into the Principles of Political Economy, [version corrigée (1780)], A. S. Skinner (éd.), Chicago, University of Chicago Press, 1966,2 vol.

Stigler George Joseph (1962), "Marshall's Principles after Guillebaud", Journal of Political Economy, vol. 70, n³, pp. 282-286.

Tanselle George Thomas (1972), "Some Principles for Editorial Apparatus", Studies in Bibliography, vol. 25, pp. 41-88.

Tanselle George Thomas (1976), "The Editorial Problem of Final Authorial Intention", Studies in Bibliography, vol. 29, pp. 167-211.

Tanselle George Thomas (1996), " Textual Instability and Editorial Idealism", Studies in Bibliography, vol. 49, pp. 1-60.

Tanselle George Thomas (2006), "Foreword", dans L. Burnard \& alii (éd.) (2006), pp. 1-6.

Tesnière Valérie (1985), "L'édition universitaire", dans R. Chartier \& H. J. Martin (1983-1986), t. III, pp. 245-255. 
Théré Christine (1998), "Economic Publishing and Authors, 1566-1789", dans G. Faccarello (éd.), Studies in the History of French Political Economy - From Bodin to Walras, London and New York, Routledge, pp. 1-56.

Théré Christine, Charles Loïc, Perrot Jean-Claude (2005a), "Introduction des éditeurs", dans F. Quesnay, Euvres économiques complètes et autres textes (1747-1769), t. I, pp. ix-xxix.

Théré Christine, Charles Loïc, Perrot Jean-Claude (2005b), "Note sur l'établissement du texte de l'édition", dans F. Quesnay, Euvres économiques complètes et autres textes (1747-1769), t. I, pp. xxx-xxxi.

Thomas d'Aquin (1266-1274), Somme théologique, Paris, Les éditions du Cerf, 1990-1996.

Todd William Burton (1951-1952), "Bibliography and the Editorial Problem in the Eighteenth Century", Studies in Bibliography, vol. 4, pp. 41-56.

Todd William Burton (1976), "The Text and Apparatus", dans A. Smith, An Inquiry into the Nature and Causes of the Wealth of Nations, R. H. Campbell, A. S. Skinner \& W. B. Todd (éd.), Oxford University Press, vol. I, pp. 61-66.

Tort Patrick (1982), "Condillac, l'économie et les signes", dans J. Sgard (éd.), Condillac et les problèmes du langage, Genève-Paris, Slatkine, pp. 421-451.

Turgot Anne-Robert Jacques, Euvres de Turgot et documents le concernant, G. Schelle (éd.), Paris, Felix Alcan, 1913-1923, 5 vol.

Vachon Stéphane (2000), " 'Pour un point, Martin perdit son âne' - L'écrivain chez son imprimeur", dans J.-L. Lebrave \& A. Grésillon (dir.), Écrire aux XVII et XVIII siècles - Genèse de textes littéraires et philosophiques, Paris, CNRS Éditions, pp. 213-240.

Vivo Giancarlo (de) (2007), "Anglo-Saxon vs Continental Schorlarship: On Critical Editions of Economic Classics", Contributions to Political Economy, vol. 26, pp. 93-102.

Vivo Giancarlo (de) (2009), "A Reply to Charles, Perrot, and Théré", Contributions to Political Economy, vol. 28, pp. 99-102.

Voltaire François-Marie Arouet (dit), Les CEuvres complètes de Voltaire, W. H. Barber \& T. Besterman (éd.), Genève, Institut et Musée Voltaire ; Oxford, The Voltaire Foundation, Paris, Touzot, 1968-.

Voltaire François-Marie Arouet (dit), "Corpus des notes marginales de Voltaire", Les Euvres complètes de Voltaire, 1979-1994, 2006-, réimpr. \& vol. 136-144.

Walker Donald Anthony (1983), "Biography and the Study of the History of Economic Thought", Research in the History of Economic Thought and Methodology, vol. 1, pp. 41-59.

Walras Léon, Correspondence of Leon Walras and related papers, W. Jaffé (éd.), Amsterdam, North-Holland, 1965, 3 vol. (vol. 1, 1857-1883 ; vol. 2, 18841897 ; vol. 3, 1898-1909).

Walras Léon \& Auguste, CEuvres Économiques Complètes, P. Dockès \& alii (éd.), Paris, Economica, 1987-2005.

Walras Léon (1874), Éléments d'économie politique pure ou Théorie de la richesse sociale, éd. définitive revue et augmentée par l'auteur [ $=5^{\mathrm{e}}$ éd.], Paris, R. 
Pichon \& R. Durand-Auzias, Lausanne, F. Rouge, 1926.

Walras Léon (1874), "Éléments d'économie politique pure ou Théorie de la richesse sociale", $5^{\text {e }}$ éd. (1926), dans A. \& L. Walras, CEuvres Économiques Complètes, vol. VIII, 1988.

Walras Léon (1874), Elements of Pure Economics, W. Jaffé (trad. de la $5^{\mathrm{e}}$ éd. : 1926), London, George Allen and Unwin, 1954.

Walras Léon (1896), "Études d'économie sociale : Théorie de la répartition de la richesse sociale", dans A. \& L. Walras, Euvres Économiques Complètes, vol. IX, 1988.

Walras Léon (1898), "Études d'économie politique appliquée : Théorie de la production de la richesse sociale", dans A. \& L. Walras, Euvres Économiques Complètes, vol. X, 1992.

Wilson Arthur MacCandless (1972), Diderot - Sa vie et son auvre, Laffont/Ramsay, 1985.

Young Warren (2005), "[Review of] D. Besomi (éd.), The Collected Interwar Papers and Correspondence of Roy Harrod", Journal of the History of Economic Thought, 27: 4, dec., pp. 459-62.

Zola Émile, Euvres complètes, H. Mitterand (éd.), Paris, Nouveau monde éd., 2002-.

\section{Sources électroniques}

(Consultables au $1^{\text {er }}$ septembre 2009)

BNF Archives et manuscrits : http://archivesetmanuscrits.bnf.fr/

Catalogue en ligne des archives et des manuscrits de l'Enseignement Supérieur: http://www.calames.abes.fr/pub/

Conference on Editorial Problems : http://www.chass.utoronto.ca/cep/

Correspondance d'Ampère : http://www.ampere.cnrs.fr/correspondance/

État sommaire des fonds d'archives privées [des Archives Nationales de France]: http://www.archivesnationales.culture.gouv.fr/chan/chan/pdf/ap/AP_etat_som maire.pdf

Euler's Correspondence : http://math.dartmouth.edu/ euler/

Gallica: http://gallica.bnf.fr/

Google Books : http://books.google.fr/

Gottfried Keller Briefwechsel : http://www.gottfriedkeller.ch/

La correspondance d'Henri Poincaré : http://www.univ-nancy2.fr/poincare/chp/

Le jardin aux sentiers qui bifurquent : http://www.taieb.net/.

Les classiques des sciences sociales : http://classiques.uqac.ca/

Manuscrits de Madame Bovary : http://bovary.univ-rouen.fr/

MEDITE : http://www-poleia.lip6.fr/ acasa/MEDITE/

Nietzsche Source : http://www.nietzschesource.org/

Project Gutenberg : http://www.gutenberg.org/

Text Archive : http://www.archive.org/details/texts 


\section{Nicolas Rieucau}

The Making of the Modern World: The Goldsmiths'-Kress Library of Economic Literature 14501850 : http://www.gale.cengage.com/DigitalCollections/products/ModernEconomy/

Wikisource : http://fr.wikisource.org/ 